\title{
Investigation into atomization spray blending property in heavy crude oil extraction under laboratory conditions
}

\author{
Jun $\mathrm{Niu}^{\mathrm{a}}$, Shuo Liu ${ }^{\mathrm{b}, \mathrm{c}, * *}$, Jing-yu $\mathrm{Xu}^{\mathrm{b}, \mathrm{c}, *}$ \\ ${ }^{\text {a }}$ Petroleum Exploration and Production Research Institute of SINOPEC, Beijing 100083, China \\ ${ }^{\mathrm{b}}$ Institute of Mechanics, Chinese Academy of Sciences, Beijing, 100190, China \\ ${ }^{\mathrm{c}}$ School of Engineering Sciences, University of Chinese Academy of Sciences, Beijing, 100049, China
}

\section{A R T I C L E I N F O}

\section{Keywords:}

Heavy crude oil extraction

Atomization spray

Blending

Viscosity reduction

Droplet behavior

\begin{abstract}
A B S T R A C T S
In this study, the atomization blending property for heavy crude oil extraction was systematically investigated both numerically and experimentally. In the experiment conducted, the blending behavior was simulated using hydraulic oil and spray with 1:10 light phase-gas atomize volume flow rate ratio in an equipment sharing the same scale as the on-site production. A Malvern Insitec droplet size analyzer was applied for the droplet size measurement, and a quick closing valve method was applied for the phase fraction measurement. A numerical simulation combined with a population balance model and the RNG $k-\varepsilon$ model was performed under both the corresponding operating conditions and a high-pressure drop environment to further investigate the blending procedure. The results show that the atomization spray blending performs better than the equivalent pure gas lifting method with more crude oil carried at the exit. Under $0.6 \mathrm{~m}^{3} / \mathrm{h}$ light phase inlet conditions, spray blending brings $8 \%$ more heavy crude than gas lifting, and the improvement rises as inlet flow rate increases. Both a droplet and liquid film are present in the casing space before blending for the spray liquid phase. In addition, the light phase droplet diameter decreases with an increase in the inlet flow rates in the lifting pipe after blending. Moreover, the blending behavior differs under various light phase inlet flow rates, while the mixture viscosity is reduced by $50 \%$ for the atomization spray method. Each of these factors was shown to be beneficial for refining the blending used in a heavy crude oil extraction technique.
\end{abstract}

\section{Introduction}

Energy consumption continues to grow, and it has been reported that energy demand worldwide will increase by more than $50 \%$ by 2030 (Shuba and Kifle, 2018). In addition, the consumption rate of petroleum is 105-times faster than the replenishment rate (Jagadevan et al., 2018). With the consumption of ordinary crude oil, heavy crude oil extraction has attracted greater attention in recent years. Owing to its density and viscosity, it is difficult to lift heavy crude oil up from a downhole environment using methods applied in an ordinary extraction. Therefore, viscosity reduction methods are essential to promoting the flow of heavy crude oil in such an environment. Consequentially, corresponding methods using heating mechanisms (Li et al., 2018a,b), additives (Subramanian et al., 2015), and blending (Jing et al., 2019) have been developed. Various heating methods including thermal and electrical heating have been surveyed and applied for a viscosity reduction. Steam-assisted gravity drainage (SAGD) is a commonly applied thermal method with a mechanism for reducing the viscosity of heavy crude oil and promoting its flow property through the heating effect induced by a high-pressure injected steam. However, considering large quantities of fresh water and cumbersome water re-cycling facilities, along with the mass production of heat required to create steam for the SAGD process, large costs are incurred under the conditions of a deep reserve. Another electrical heating method has been under development for decades with numerous studies conducted and site application records available (Rangel-German et al., 2004). In terms of additives, numerous chemicals have been applied as additives to promote the decrease in viscosity of heavy crude oil. To date, polymers that reduce the oil-water surface tension (Du and Guan, 2004; Gao, 2011) and alkaline chemicals that react with the surface-active components originated from oil (Guo et al., 2016) have been applied in various reservoirs around the world. As with a blending method, water, light oil, hydrocarbon gases, and alcohol (Ghannam et al., 2012) have been applied to mix with the heavy crude oil to obtain a suspension or mixture with an

\footnotetext{
* Corresponding author. Institute of Mechanics, Chinese Academy of Sciences, Beijing, 100190, China.

** Corresponding author. Institute of Mechanics, Chinese Academy of Sciences, Beijing, 100190, China.

E-mail addresses: liushuo@imech.ac.cn (S. Liu), xujingyu@imech.ac.cn (J.-y. Xu).
} 


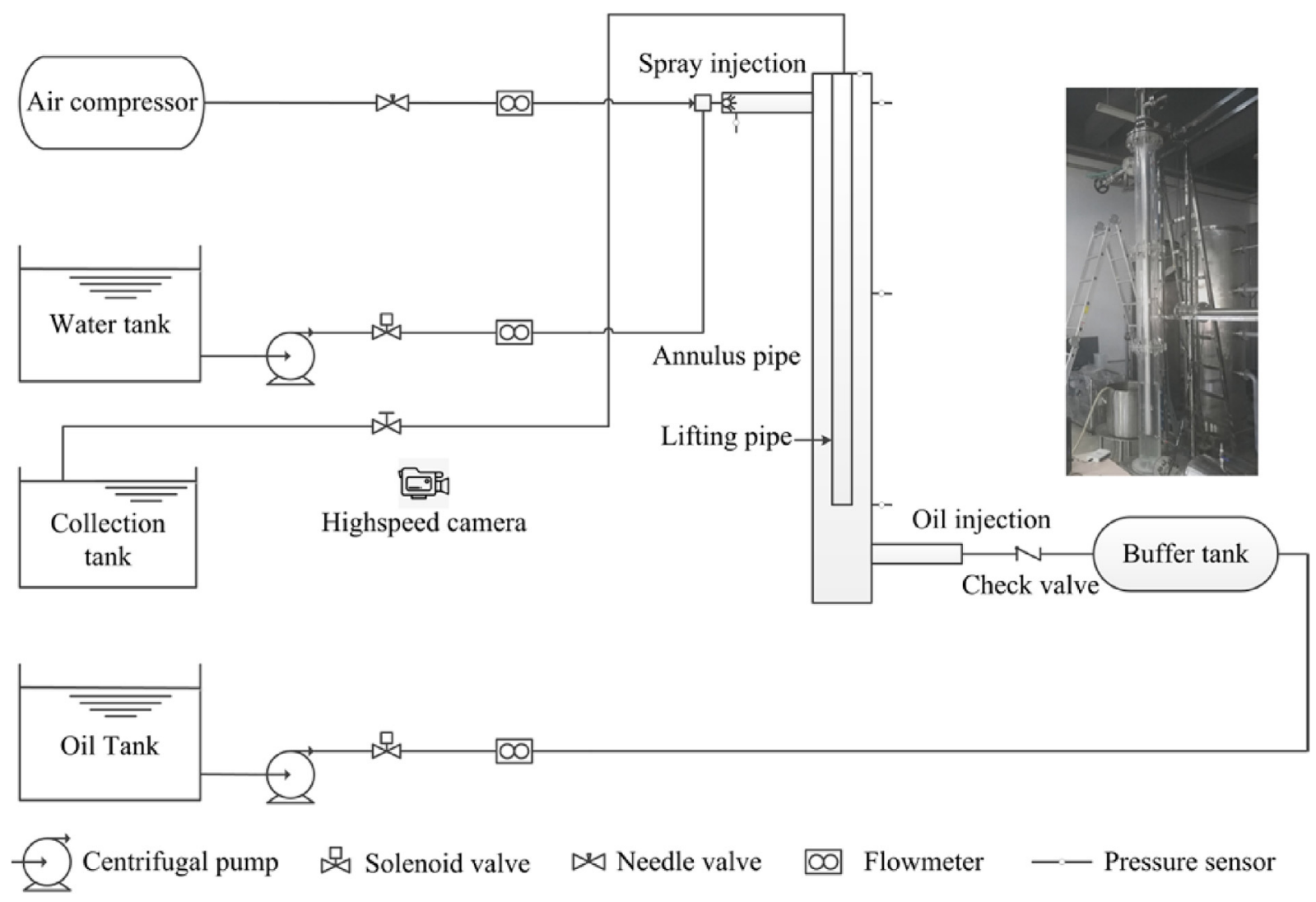

Fig. 1. The whole experiment equipment.

Table 1

Physical property parameters of fluids in the experiment and simulation (ambient temperature).

\begin{tabular}{llll}
\hline & Gas & Liquid 1 & Liquid 2 \\
\hline Fluids & air & water & hydraulic oil \\
Density $\left(\mathrm{kg} / \mathrm{m}^{3}\right)$ & 1.225 & 998 & 900 \\
Viscosity $(\mathrm{mPa} \cdot \mathrm{s})$ & 0.00179 & 1 & 116 \\
Surface tension $(\mathrm{N} / \mathrm{m})$ & $\sigma_{\text {air } / \text { water }}=0.073$ & $\sigma_{\text {oil } / \text { water }}=0.026$ & $\sigma_{\text {air } / \text { oil }}=0.062$ \\
\hline
\end{tabular}

ideal mobility for a lifting operation from a well.

Although the methods above may be combined (Jing et al., 2019) on-site to achieve a better viscosity, each method alone should be investigated thoroughly prior to refinement and combination with other methods. In terms of blending, despite being the oldest and most preferred choice for a heavy crude oil viscosity reduction, this method still has challenging properties when considering that the composition of oil may affect the required oil ratio. Researchers have recently worked on the rheology and viscosity properties of blended heavy crude oil (Martinez-Palou et al., 2011). Hasan et al. (2010) surveyed the rheological properties of heavy crude and its mixture with light crude experimentally, determining that the heavy crude has a 0.7 Pa yield stress, with no corresponding reports made on heavy-light crude mixtures. Through offline rheometer measurements, Ghannam et al. (2012) found that the storage and loss moduli of heavy crude oil decrease significantly by the addition of $10 \%$ light crude oil. Jing et al. (2019) provided a model predicting the viscosity and contact angle of watery heavy crude oil blended with light oil.

Despite research in two the mixture rheology properties described above, methods for the blending and mixture-lifting of heavy crude oil extraction are also important. For non-flowing heavy crude oil wells, a gas-lift can be applied using research conducted by Guet and Ooms (2006). In addition, blending can be applied either up or down the pump, and the light phase is always injected in the form of a continuous fluid (Yang, 2017). To obtain a better mixture and promote lifting, light phase droplets distributed during gas injection are an ideal choice. Under this condition, spray atomization is essential in creating light oil sprays. For decades, researchers have conducted numerous studies on the droplet size distribution immediately after atomizing the nozzle, and corresponding theoretical prediction models have been proposed (Wu et al., 2006; Luo et al., 2014; Wang et al., 2017; Xia et al., 2017). Although the studies above provide a droplet size distribution, studies on light phase droplet blending properties with heavy crude oil remain rare in literature. Similar studies have mostly focused on the spray combustion of engines. Consequently, investigations into the atomization blending properties between a heavy crude oil and a light phase spray with a site scale flow domain are essential. In this study, a systematic laboratory experiment and a numerical simulation on the atomization blending behavior between heavy crude oil and a light liquid phase, together with gas lifting, were conducted, which can provide a reference for heavy crude extraction.

\section{Experiment design}

\subsection{Dimensionless analysis}

A flow experiment is essential when considering the intricate mechanism of atomized blending between a heavy crude oil and a spray. Prior to conducting an experiment, a dimensionless analysis of the testing system is essential.

Owing to the scale effect properties of a multiphase flow, experiment models on fractions of the site production wells may bring about a flow type variance under similar operating conditions. Thus, the experiment system applied in this study shares the same scale of geometry with field production wells. As shown in Fig. 1, the blending equipment is made up of an annulus casing pipe and a steel lifting pipe to simulate 

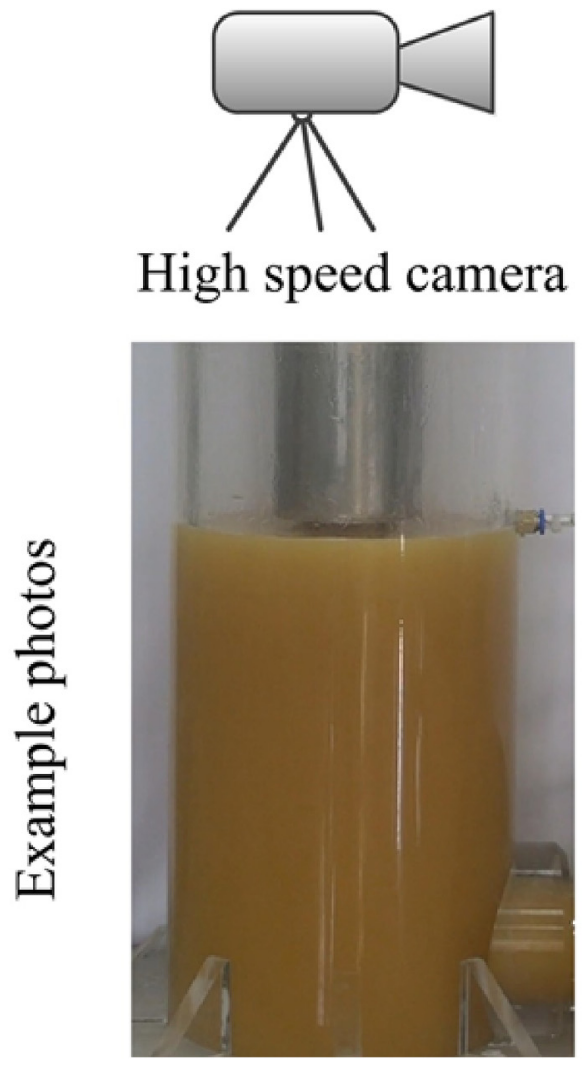
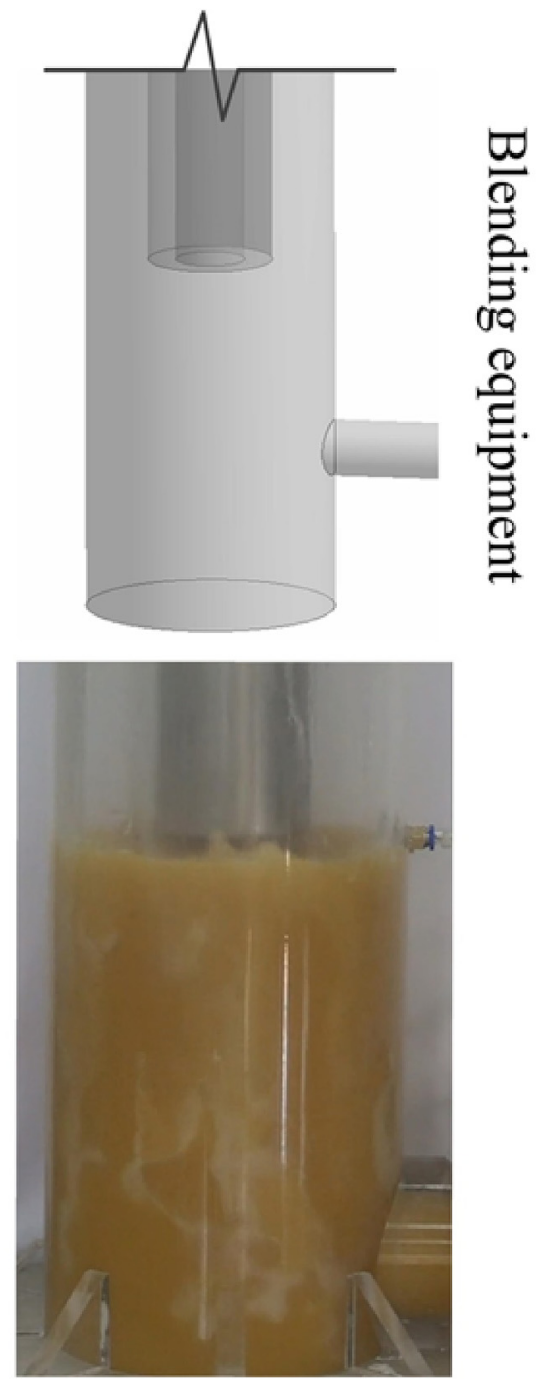

Fig. 2. Example photos of high speed camera.

the down-hole blending procedure. Their inner diameters are 180 and $50 \mathrm{~mm}$, respectively, and the thickness of the lifting pipe is $20 \mathrm{~mm}$. Hence, the outer and inner diameters of the annulus space are 180 and $90 \mathrm{~mm}$, respectively. To the height of the blending equipment, an $H / d$ value (where $H$ is the height of the equipment and $d$ is the equivalent diameter of the annulus pipe) of larger than 35 is sufficient for spray droplet size development. The droplet size distribution can be relatively stable under this condition. Hence, the height of the equipment is $3.16 \mathrm{~m}$.

For a spray injected in the equipment, using a Malvern Insitec droplet size analyzer, the droplet ranges from 20 to $2000 \mu \mathrm{m}$ in size. The spray entrance Weber number, which characterizes the relationship between the inertia force and interfacial tension, can be obtained based on the operating conditions described in Section 2.5, and the atomized nozzle geometry parameter is given through Eq. (1):

$W e=\frac{\rho_{a} u_{a / w}^{2} d_{w}}{\sigma_{\text {air } / \text { water }}}$,

where $\rho_{a}$ is the density of the air phase, $u_{a / w}$ is the relative velocity between the air and droplets, $d_{w}$ is the diameter of the water droplets, and $\sigma_{\text {air/water }}$ is the interfacial tension. In this study, the entrance spray droplet Weber number ranges from 0.28 to 4.4 .

The simulated Reynolds number of the heavy crude oil phase can be defined using Eq. (2), where $\rho_{o}$ is the density of the oil phase, $u_{o}$ is the entrance velocity of the oil phase at the oil injection nozzle, $d_{o}$ is the characteristic length of the oil injection nozzle, and $\mu_{o}$ is the viscosity of the oil phase. The values of these parameters are provided in Table 1 and described in Section 2.5. Hence, the Reynolds number of the oil phase ranges from 1,396 to 7,758 .

$R e=\frac{\rho_{o} u_{o} d_{o}}{\mu_{o}}$

The viscosity ratio $\lambda$ (defined by $\mu_{1} / \mu_{2}$, where $\mu$ is the viscosity) is an important dimensionless parameter when discussing the phase blending. Based on the material properties in Table 1, the viscosity ratio of oil to water $\lambda_{o / w}$ is 116 , and the viscosity ratio of water to air $\lambda_{w / a}$ is 559.

\subsection{Experiment system}

The experiment was conducted on a multiphase simulation system at the Institute of Mechanics, Chinese Academy of Sciences. The blending equipment described above has two entrances. The entrance at the top of the equipment was set for a spray injection. As shown in Fig. 1, the water phase from a water tank was atomized by air from an air compressor at the atomizer and injected into the blending equipment. The entrance at the bottom was set for oil injection and connected with a buffer tank and a check valve to stabilize the oil injection uniformly. The exit of the blending equipment was mounted at the top of the lifting pipe and connected with the collection tank. Based on the 


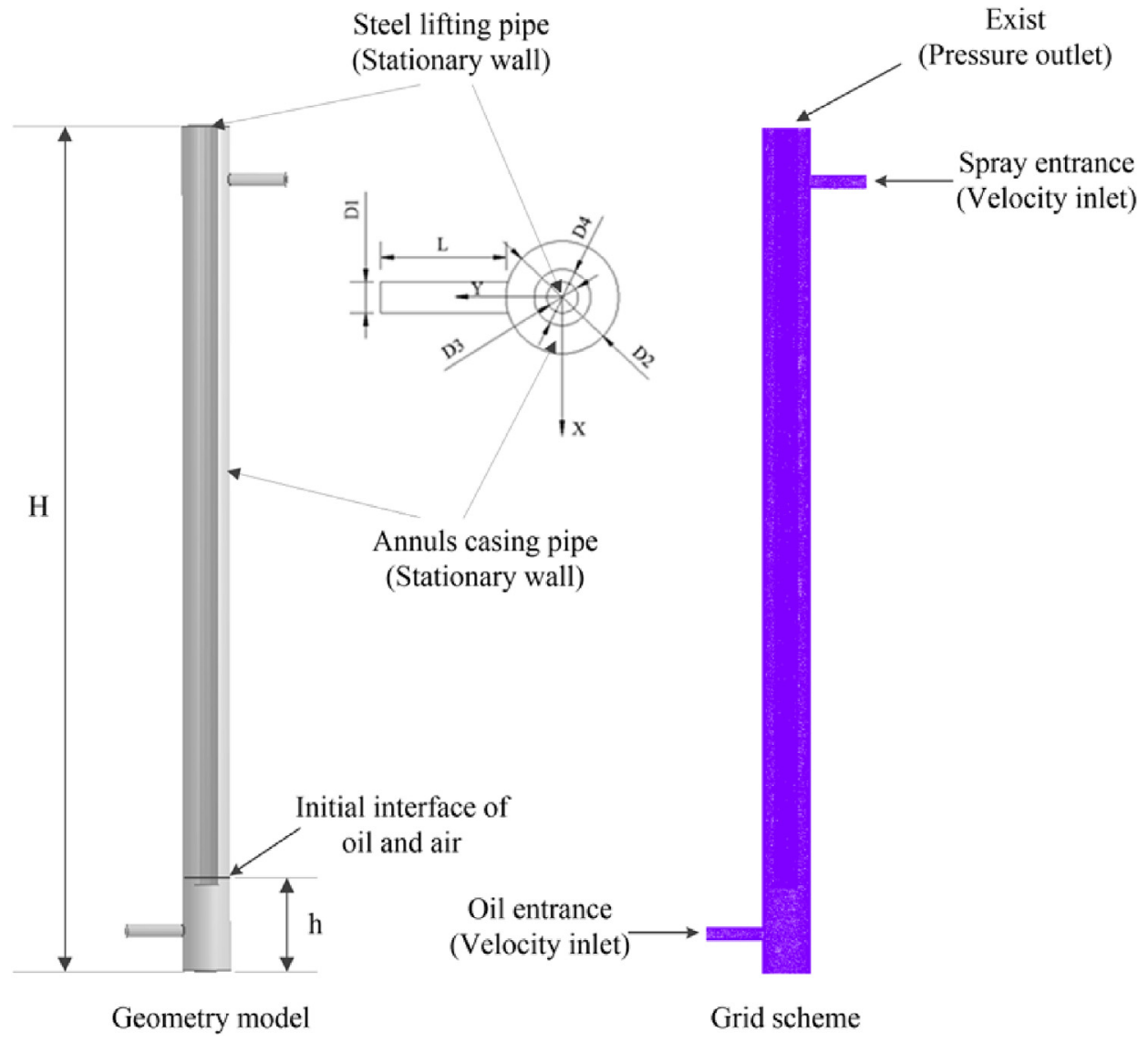

Fig. 3. Geometry model, grid scheme and boundary conditions.

Table 2

The dimension of geometrical structure parameters.

\begin{tabular}{lll}
\hline Geometrical structure parameters & Unit & Dimension \\
\hline The inlet diameter of model, $D 1$ & $\mathrm{~mm}$ & 50 \\
Annulus tube outer diameter, $D 2$ & $\mathrm{~mm}$ & 180 \\
The outlet of model, $D 3$ & $\mathrm{~mm}$ & 50 \\
Annulus tube inner diameter, $D 4$ & $\mathrm{~mm}$ & 90 \\
The total height, $H$ & $\mathrm{~mm}$ & 3160 \\
Nozzle length, $L$ & $\mathrm{~mm}$ & 200 \\
Initial oil length, $h$ & $\mathrm{~mm}$ & 580 \\
\hline
\end{tabular}

flow loop, atomization of the blending property between the heavy oil phase and lighter phase was thoroughly investigated.

\subsection{Measuring system and error analysis}

The aim of the experiment research was to investigate the blending property and provide the boundary conditions of the numerical simulation. Hence, the inlet droplet size distribution, flow rate, and pressure in the annulus casing pipe are essential. Correspondingly, a Malvern Insitec droplet size analyzer for a droplet size distribution

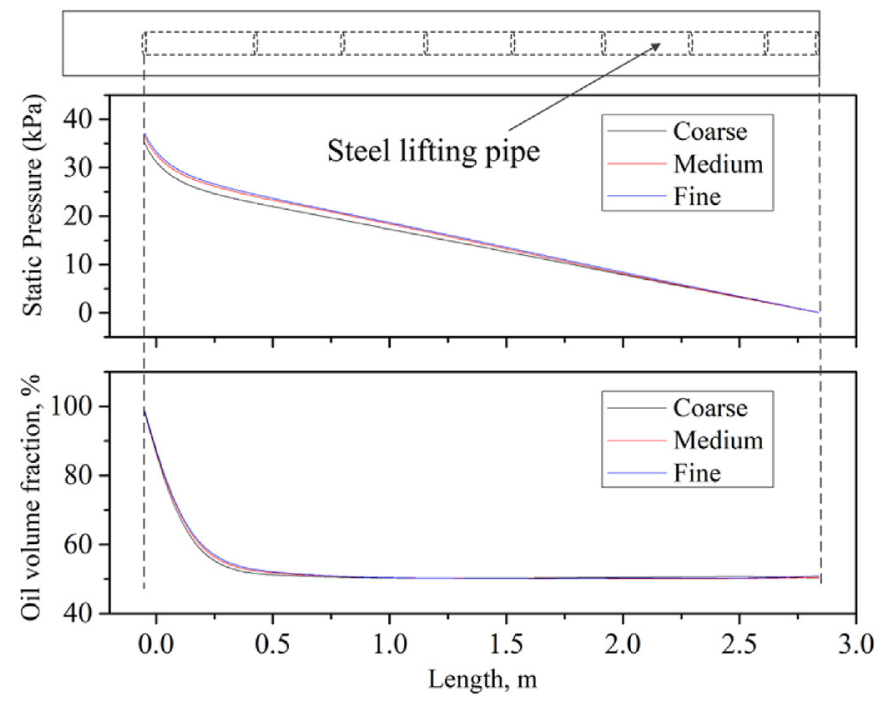

Fig. 4. Section average pressure and oil volume fraction distribution along steel lifting pipe for various grid schemes.

Table 3

Boundary condition settings.

\begin{tabular}{|c|c|c|c|}
\hline No & Geometry & Boundary condition & Remarks \\
\hline 1 & Spray entrance & Velocity inlet & Air phase velocity, water phase velocity, phase fraction, water droplet size distribution \\
\hline 2 & Oil entrance & Velocity inlet & Oil phase velocity \\
\hline 3 & Exit & Pressure outlet & - \\
\hline 4 & Annulus casing wall & Stationary wall & Scable wall function \\
\hline 5 & Steel lifting pipe & Stationary wall & Scable wall function \\
\hline
\end{tabular}


Table 4

Character droplet size of each bin.

\begin{tabular}{ll}
\hline Number & Size, mm \\
\hline Bin-0 & 34.756 \\
Bin-1 & 12.288 \\
Bin-2 & 4.344 \\
Bin-3 & 1.536 \\
Bin-4 & 0.543 \\
Bin-5 & 0.192 \\
Bin-6 & 0.068 \\
Bin-7 & 0.024 \\
Bin-8 & 0.008 \\
Bin-9 & 0.003 \\
\hline
\end{tabular}

measurement, a Honeywell pressure sensor for a pressure measurement, a Krohen Optiflux400 for a liquid flow rate measurement, and a thermal mass flowmeter for measurement of the gas phase were applied in this study. Moreover, a high-speed camera was applied to record the image data of inside the casing. The high-speed camera used was a SONY HXR-MC2500. Example photographs of the high-speed camera are shown in Fig. 2, which present both the entire range of the testing section and local parts including the upper section and bottom of the casing pipe. Fig. 1 provides a distribution of all measurement equipment mentioned above.

In terms of an error analysis, systematic errors should first be mentioned. Hirleman et al. (1984) found that both detector calibration errors and nonideal lens effects result in up to $15 \%$ of the variations in the instrument response for Malvern Insitec droplet size analyzer. When applying a Honeywell pressure sensor, after calibration, the systematic error according to the instructions reaches $0.15 \%$. For Rosemont flow meters, the systematic error is $0.1 \%$. To avoid an accidental error, the measurement procedures are repeated several times for each operating condition.

\subsection{Working fluids}

The experiment applied pure water and anti-wear hydraulic oil (oil for abbreviation) to simulate the light phase and heavy crude oils, respectively. Moreover, air was applied to produce the spray droplets. The anti-wear hydraulic oil (L-H M46) was produced by Shengkang Chemical Ltd., Co. in Hebei, China. When operated under indoor temperatures of $25^{\circ} \mathrm{C}$, the properties of the working fluids are as listed in Table 1.

\subsection{Operating conditions}

The oil flow rate applied during the experiment ranged $3.6-20.0 \mathrm{~m}^{3} / \mathrm{h}$. According to a study by Ghannam et al. (2012) work, a $10 \%$ additional light phase can achieve the ideal viscosity reduction performance. Hence, the corresponding water flow rate ranges from 0.36 to $2.0 \mathrm{~m}^{3} / \mathrm{h}$. The water was turned into a spray using an air-blastatomizer. In total, the operating conditions were as follows: a 3.6, 6.0, $8.4,10.8,15.0$, and $20.0 \mathrm{~m}^{3} / \mathrm{h}$ gas inlet flow rate coupled with a water inlet flow rate of $0.36,0.6,0.84,1.08,1.5$, and $2.0 \mathrm{~m}^{3} / \mathrm{h}$ at the atomizer entrance. In each case, the data matrix contains not only the entrance droplet distribution, but also the phase flow rates and pressure. Moreover, a gas lifting without a spray in the corresponding inlet flow rates was conducted for contrast.

\section{Numerical simulation}

\subsection{Mathematical models}

Although a flow experiment can characterize the atomized blending phenomenon, a numerical simulation is still essential for supplementation of the detailed blending properties. The essence of the numerical simulation applied in this study is to solve the air-water-oil three-phase Navier-Stokes equations. An Eulerian multiphase model was applied for its ability to simulate separate and interacting phases.

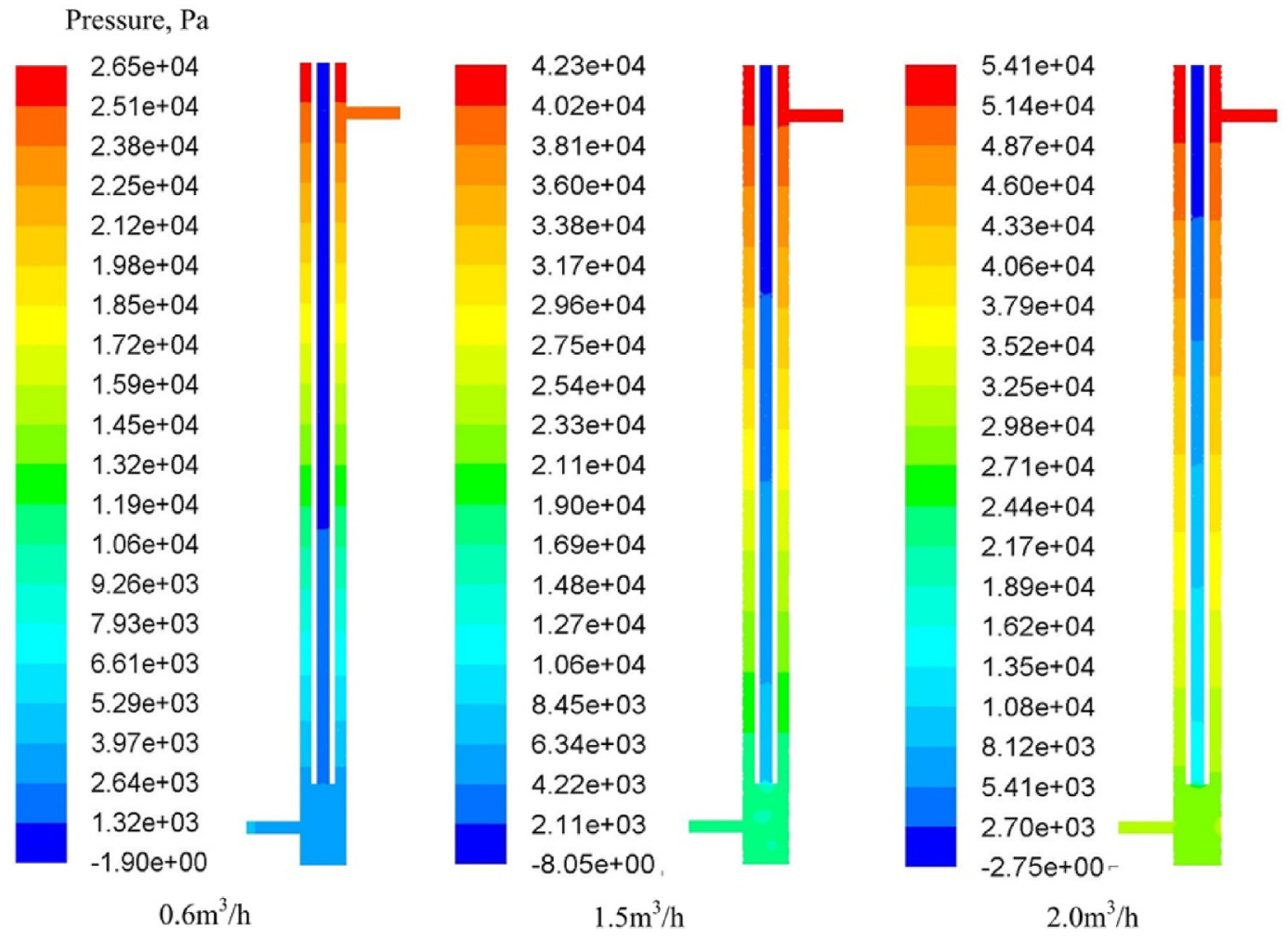

Fig. 5. Pressure distribution for various operating conditions characterized by inlet water flow rates. 
Oil phase velocity magnitude, $\mathrm{m} / \mathrm{s}$
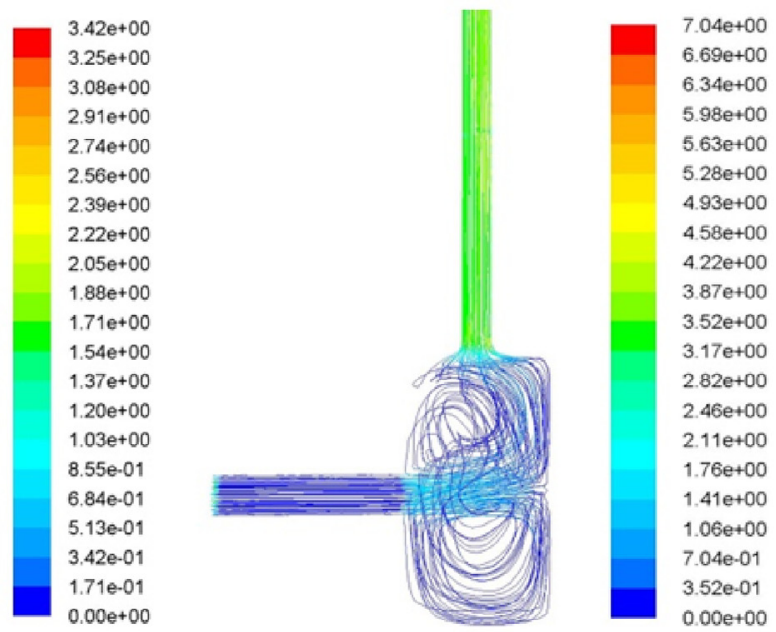

$0.6 \mathrm{~m}^{3} / \mathrm{h}$

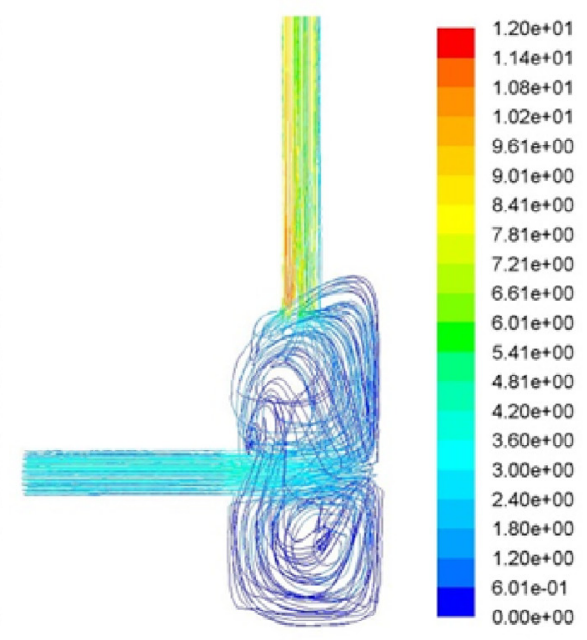

$1.5 \mathrm{~m}^{3} / \mathrm{h}$

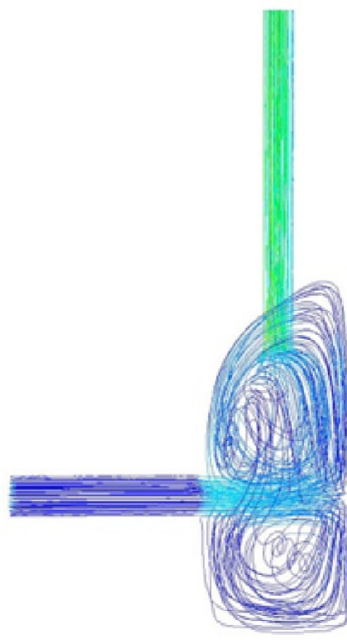

$2.0 \mathrm{~m}^{3} / \mathrm{h}$

Fig. 6. Path line distribution for various operating conditions.

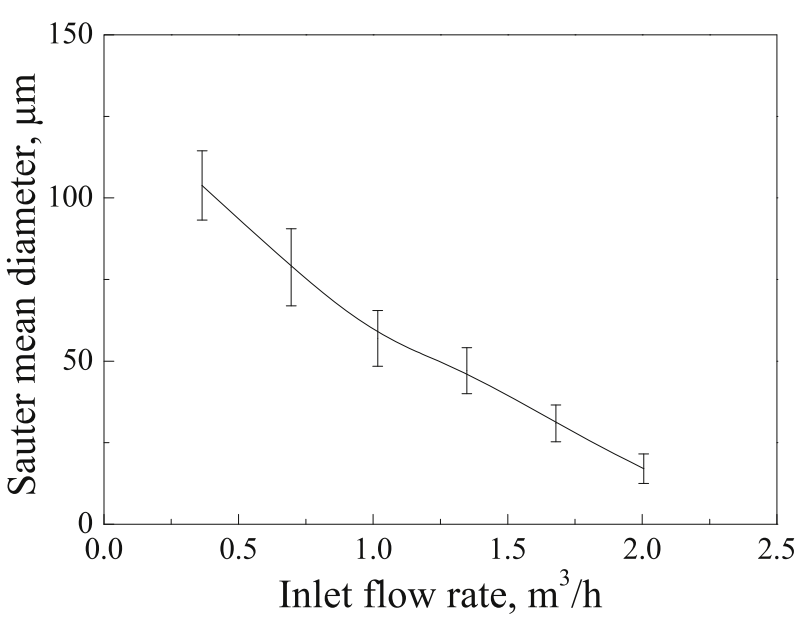

Fig. 7. Water droplets Sauter mean diameter variation right at the nozzle.

Eqs. (3)-(5) provide mathematical equations of the Eulerian multiphase model.

$\sum_{q=1}^{n} \alpha_{q}=1$

$\frac{\partial\left(\alpha_{q} \rho_{q}\right)}{\partial t}+\nabla \cdot\left(\alpha_{q} \rho_{q} \vec{v}_{q}\right)=\sum_{p=1}^{m}\left(\dot{m}_{p q}-\dot{m}_{q p}\right)+S_{q}$

$\frac{\partial}{\partial t}\left(\alpha_{q} \rho_{q} \overrightarrow{v_{q}}\right)+\nabla \cdot\left(\alpha_{q} \rho_{q} \vec{v}_{q} \overrightarrow{v_{q}}\right)=-\alpha_{q} \nabla p+\nabla \cdot \overline{\overline{\tau_{q}}}+\alpha_{q} \rho_{q} \vec{g}+$

$\sum_{p=1}^{n}\left(\vec{R}_{p q}+\dot{m}_{p q} \vec{v}_{p q}-\dot{m}_{q p} \vec{v}_{q p}\right)+\left(\vec{F}_{q}+\vec{F}_{l i f t, q}+\vec{F}_{v m, q}\right)$

In the equations, $q$ refers to the $q$ th phase, $v_{q}$ is the velocity of phase $q, \dot{m}_{p q}$ is the mass transfer from the $p$ th phase to the $q$ th phase, $\dot{m}_{q p}$ is the mass transfer from the $q$ th phase to the $p$ th phase, and $S_{q}$ is the source term. Based on the circumstances of this study, the mass transfer among phases can be neglected. The same is true for the source term. Here, $\overline{\overline{\tau_{q}}}$ is the $q$ th phase of the stress-strain tensor, $\vec{F}_{q}$ is the body force, $\vec{F}_{l i f t, q}$ is the lift force, and $\vec{F}_{v m, q}$ is the virtual mass force.

Moreover, as the water entrance fraction is less than $10 \%$ considering all phases together, and with the blending properties between oil and water being the main focus of study, the water droplet behavior, including the size distribution and size change, should be included. A population balance equation for the water phase was added in the equations above. In the absence of evaporation or dissolution, the population balance equation is shown in Eq. (6) (Liao and Lucas, 2009). Here, $n(V i, x, t)$ is the droplet number density, and $S_{b}$ and $S_{c}$ are the source terms resulting from a droplet breakup and coalescence. Reliable models of the droplet breakup and coalescence used to represent the source terms $S_{b}$ and $S_{c}$, respectively, can alleviate this problem. In this study, Luo's model for kernel breakup and kernel coalescence was applied.

$\frac{\partial n\left(V_{i}, x, t\right)}{\partial t}+\nabla x\left[u\left(V_{i}, x, t\right) \cdot n\left(V_{i}, x, t\right)\right]=S_{b}+S_{c}$

In the turbulent model selection, based on an experiment observation, a relative weak vortex exists owing to the presence of an inlet branch and a steel pipe inside the casing. Under this condition, the RNG $k-\varepsilon$ model is an ideal choice, and was derived using a rigorous statistical technique. Although it shares a similar form with the $k-\varepsilon$ model, it includes refinements on the swirling flows and provides an analytical formula for a turbulent flow. The equation of the RNG $k-\varepsilon$ model is shown in Eqs. (7) and (8). Here, $G_{k}$ represents the generation of turbulence kinetic energy owing to the mean velocity gradients, $G_{b}$ is the generation of turbulence kinetic energy from buoyancy, and $Y_{M}$ represents the contribution of the fluctuating dilatation in an incompressible turbulence to the overall dissipation rate.

$$
\begin{aligned}
\frac{\partial}{\partial t}(\rho k)+\frac{\partial}{\partial x_{j}}\left(\rho k u_{i}\right)= & \frac{\partial}{\partial x_{j}}\left(\alpha_{k} \mu_{e f f} \frac{\partial k}{\partial x_{j}}\right)+G_{k}+G_{b}-\rho \varepsilon-Y_{M}+S_{K} \\
\frac{\partial}{\partial t}(\rho \varepsilon)+\frac{\partial}{\partial x_{j}}\left(\rho \varepsilon u_{i}\right)= & \frac{\partial}{\partial x_{j}}\left(\alpha_{\varepsilon} \mu_{e f f} \frac{\partial \varepsilon}{\partial x_{j}}\right)+G_{1 \varepsilon} \frac{\varepsilon}{k}\left(G_{k}+G_{3 \varepsilon} G_{b}\right)-G_{2 \varepsilon} \rho \frac{\varepsilon^{2}}{k} \\
& -R_{\varepsilon}+S_{\varepsilon}
\end{aligned}
$$

\subsection{Geometric model and boundary conditions}

Prior to the grid generation, a geometry model of the fluid domain should first be constructed. Fig. 3 shows the geometrical outline of the fluid domain, and the detailed dimensional parameters are provided in Table 2. The main body of the model is a $180 \mathrm{~mm}$ diameter solid cylinder with a hollowed-out steel pipe. The inner and outer diameters of the steel pipe are 50 and $90 \mathrm{~mm}$, respectively. Near the top of the main body, a nozzle vertical to the cylinder axis is added for injection of air and droplets. At the bottom of the cylinder, a horizontal tube is also 


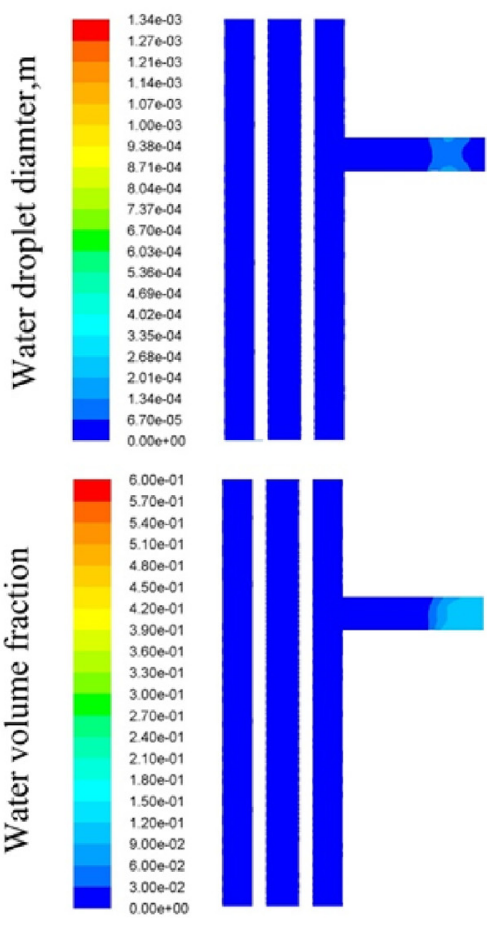

$0.1 \mathrm{~s}$
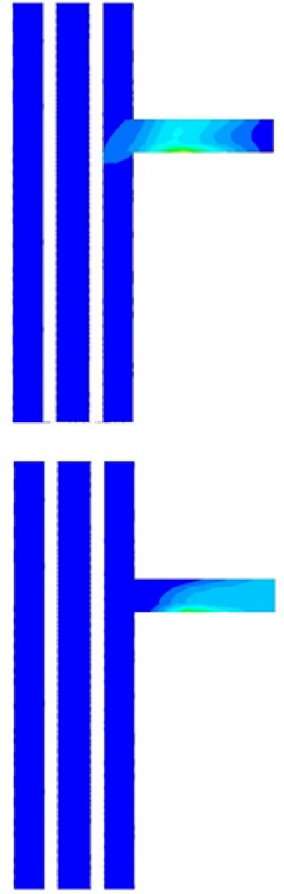

$0.15 \mathrm{~s}$
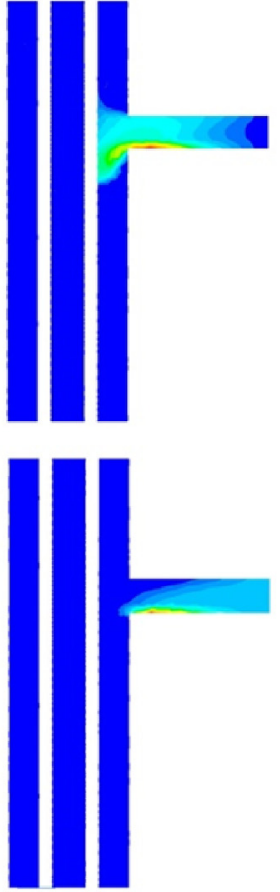

$0.2 \mathrm{~s}$

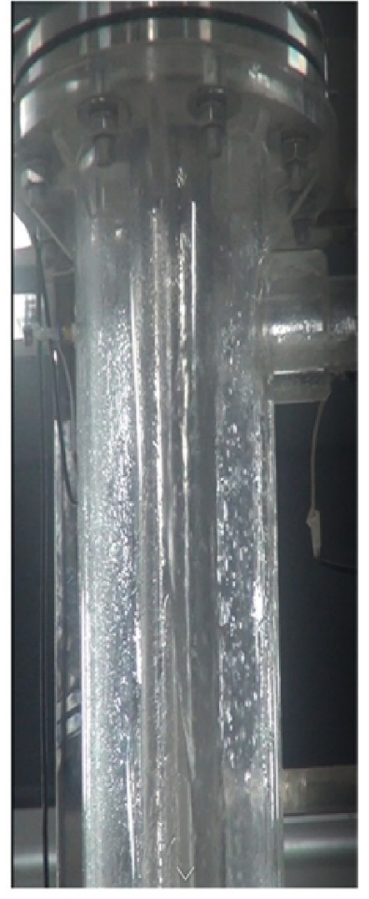

Fig. 8. Water droplet size and volume fraction distribution near spray injection nozzle at $1.5 \mathrm{~m}^{3} / \mathrm{h}$ inlet flow rate.

Water droplet size, $\mathrm{m}$

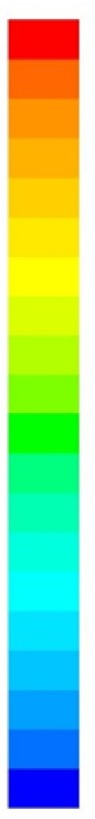

$$
\begin{aligned}
& 1.11 \mathrm{e}-02 \\
& 1.05 \mathrm{e}-02 \\
& 9.97 \mathrm{e}-03 \\
& 9.41 \mathrm{e}-03 \\
& 8.86 \mathrm{e}-03 \\
& 8.31 \mathrm{e}-03 \\
& 7.75 \mathrm{e}-03 \\
& 7.20 \mathrm{e}-03 \\
& 6.64 \mathrm{e}-03 \\
& 6.09 \mathrm{e}-03 \\
& 5.54 \mathrm{e}-03 \\
& 4.98 \mathrm{e}-03 \\
& 4.43 \mathrm{e}-03 \\
& 3.88 \mathrm{e}-03 \\
& 3.32 \mathrm{e}-03 \\
& 2.77 \mathrm{e}-03 \\
& 2.22 \mathrm{e}-03 \\
& 1.66 \mathrm{e}-03 \\
& 1.11 \mathrm{e}-03 \\
& 5.56 \mathrm{e}-04 \\
& 3.00 \mathrm{e}-06
\end{aligned}
$$

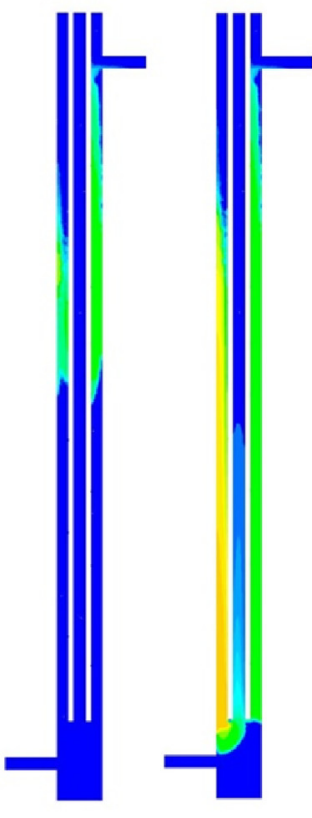

$0.6 \mathrm{~s}$

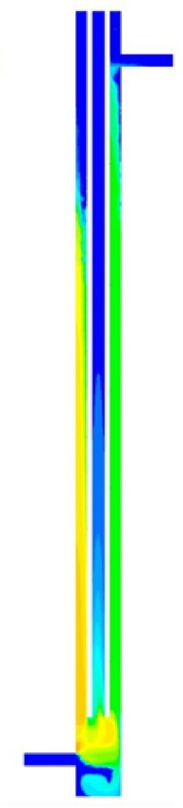

$1.8 \mathrm{~s}$
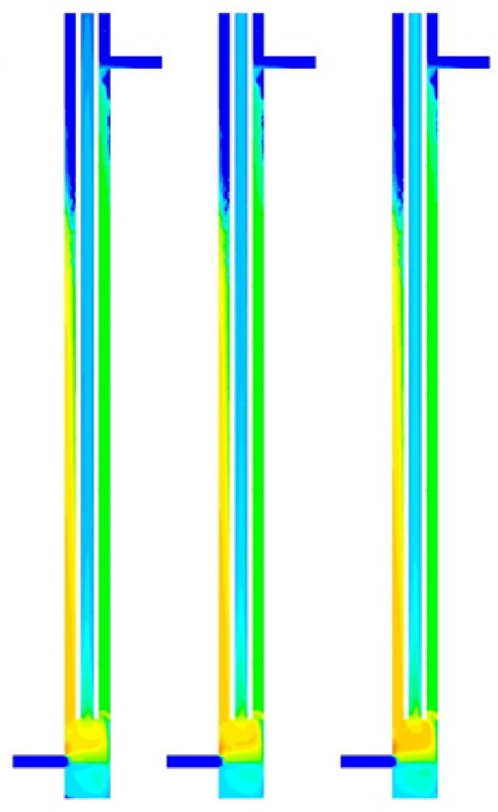

$2.4 \mathrm{~s}$

$3.0 \mathrm{~s}$

$3.6 \mathrm{~s}$

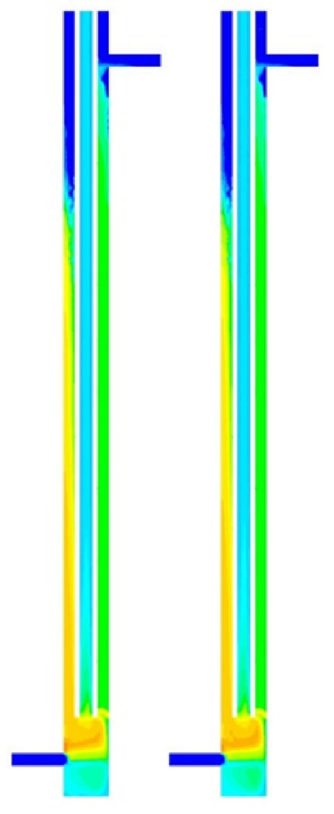

$4.2 \mathrm{~s}$

$4.8 \mathrm{~s}$

Fig. 9. Water droplet size distribution for $1.5 \mathrm{~m}^{3} / \mathrm{h}$ inlet flow rate under different time.

added for the entrance of the oil phase.

For the boundary condition, the spray and oil injection entrances are set as the velocity inlet, whereas the top of the steel pipe is set as the pressure outlet. Specially, for the spray injection entrance, the water droplet size distribution originates from the Malvern Insitec droplet size analyzer measurement. A scalable wall function is applied for the Plexiglass inner wall and steel surface. All boundary conditions are as shown in Table 3.

\subsection{Grid generation and independent study}

The numerical simulation was conducted on software Fluent. Using the ANSYS workbench mesh generator, tetrahedron grids of different densities were created based on the geometry of the fluid domain. In a 


\section{Water droplet size, $\mathrm{m}$}

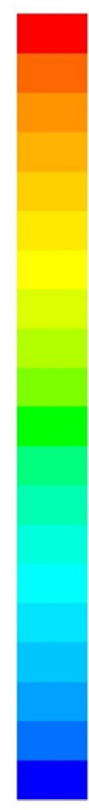

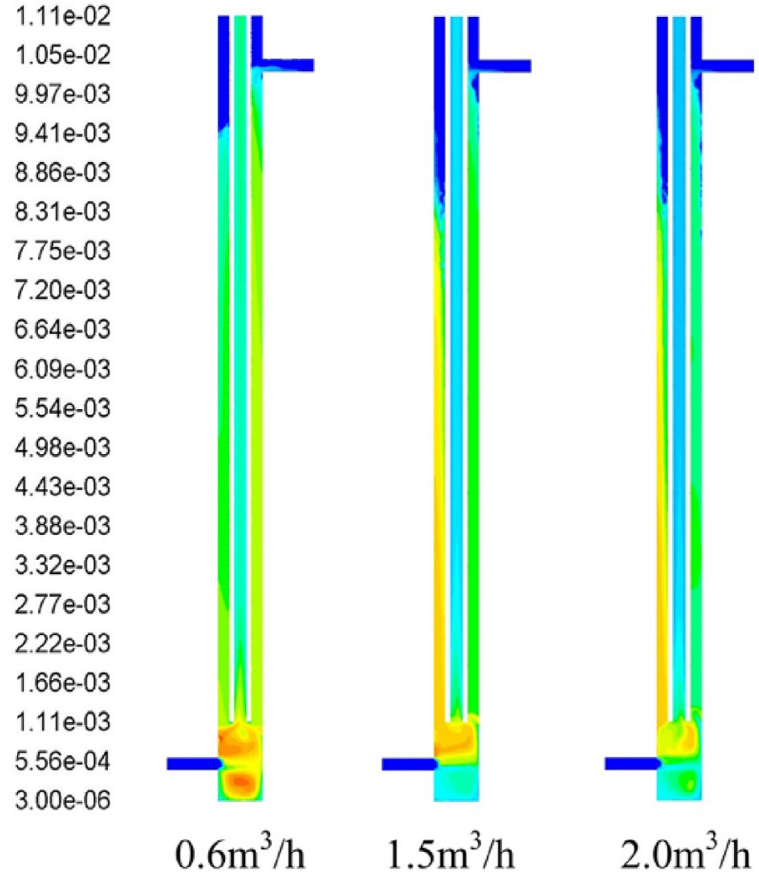

Fig. 10. Water droplet size distribution in the whole flow field for various inlet flow rates.

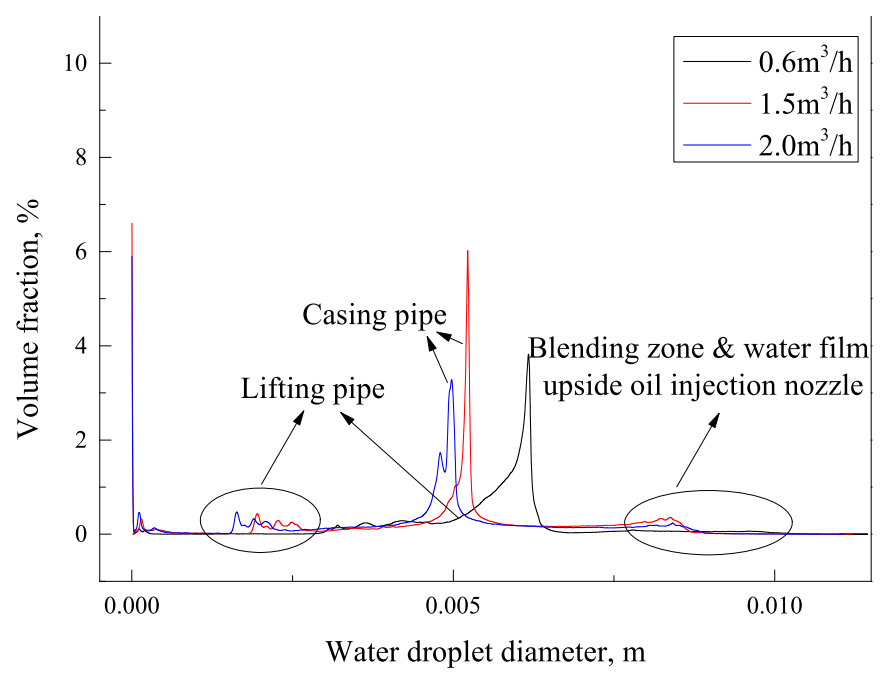

Fig. 11. Water droplet size distribution in the whole flow field for various entrance flow rates.

grid-independent study, three grid schemes were proposed, namely, a coarse scheme with 645,627 cells, a medium scheme with $1,419,917$ cells, and a fine scheme with $1,898,423$ grids. A gas lifting oil condition with a $15 \mathrm{~m}^{3} / \mathrm{h}$ inlet flow rate was chosen to conduct a gridindependent study. The sectional average pressure and variation in the oil phase fraction along the steel-lifting pipe were selected as the reference parameters. Fig. 4 illustrates the sectional average pressure and oil volume fraction changes along the steel-lifting pipe. As shown in Fig. 4, in terms of the sectional average pressure, the deviation from the medium scheme to the coarse scheme is $5.2 \%$, whereas the deviation from the fine scheme to the medium scheme is $2.0 \%$. In terms of the sectional average pressure, the deviation from the medium scheme to the coarse scheme is $1.0 \%$, whereas the deviation from the fine scheme to the medium scheme is $0.46 \%$. In terms of both the sectional average pressure and oil volume fraction, the discrepancy between the coarse scheme and the medium scheme is far less than the discrepancy between the medium and fine schemes. The medium scheme is sufficiently fine for a numerical simulation.

\subsection{Solution settings}

Based on the physical properties and mathematical models discussed above, an Eulerian multiphase model and a PBM model are applied. Considering oil as the main phase at the bottom of the fluid domain and the blending behavior as a concern of this study, the primary phase was defined as oil, whereas air and water were considered secondary phases. Considering the possible water droplet sizes, 11 bins ranging from $3 \mu \mathrm{m}$ to $3 \mathrm{~cm}$ were created using a discrete method for the water phase PBM setting, and the details of each bin size are as shown in Table 4.

Similar to the solution settings, considering that the fluid domain is non-stationary, a transient behavior is selected with a time step of $1.5 \mathrm{~ms}$. Moreover, for the initial condition, oil was patched for fields under a steel pipe, and above the oil, pure air without water droplets was initially present. The initial air-oil interface can be seen in Fig. 2 . Phase-coupled SIMPLE was chosen as the pressure-velocity coupling scheme. Spatial discretization was set to the high accuracy form and the residual scales were set to $10^{-6}$.

\section{Results and discussion}

\subsection{Flow domain characters}

Prior to the investigation into the droplet size distribution and blending properties, the general flow characters of the flow domain should first be clarified for further discussion. Herein, the pressure distribution and pathline are of primary importance. Fig. 5 shows the pressure distribution for the experimental flow domain under specific operating conditions after the flow domain stabled. In the figure, the red color represents high pressure whereas the blue color represents low pressure with other colors as the transition. As one more aspect requiring clarification, among the various operating conditions, a pressure of relatively zero was set at the exit of the steel lifting pipe for convenience of comparison. It can be seen in the experimental operating conditions that the pressure decrease ranges from 26 to $54 \mathrm{kPa}$ and increases with an increase in the inlet flow rate. For all the operating conditions, pressure from top of annuls casing to exist of lifting pipe decrease gradually. As inlet flow rate rises, pressure difference between entrance and exist of lifting pipe becomes more obvious, this can be attributed to influence velocity distribution.

As shown in Fig. 6, the oil phase velocity and pathline vary according to the inlet flow rates. After the oil is injected into the oil entrance, it hits the walls on the right side with diffusion along the path. A circular flow resulting from a diffusion occurred at the half point and hindered the wall on the right side, filling the flow domain to obtain a mixture of air and water phases, and then gathered at the steel lifting pipe. The larger the inlet flow rate, the higher the invasion of the upper side of the initial oil-air interface in the casing space.

\subsection{Droplet size distribution}

As water moves in the form of discrete droplets within the domain, its flow behavior is influenced by its size, thus affecting the blending properties with heavy crude oil. In addition, the water droplet size distribution immediately after the air-blast-atomizer is the boundary condition of the flow domain. Fig. 7 shows the Sauter mean diameter of the exit of the atomizer with error bar of the Malvern Insitec droplet size analyzer measurement. Here, the Sauter mean diameter can be defined through Eq. (9), where $d_{i}$ is the ith droplet diameter, and characterizes the energy properties of the particles and has a 
Water volume fraction
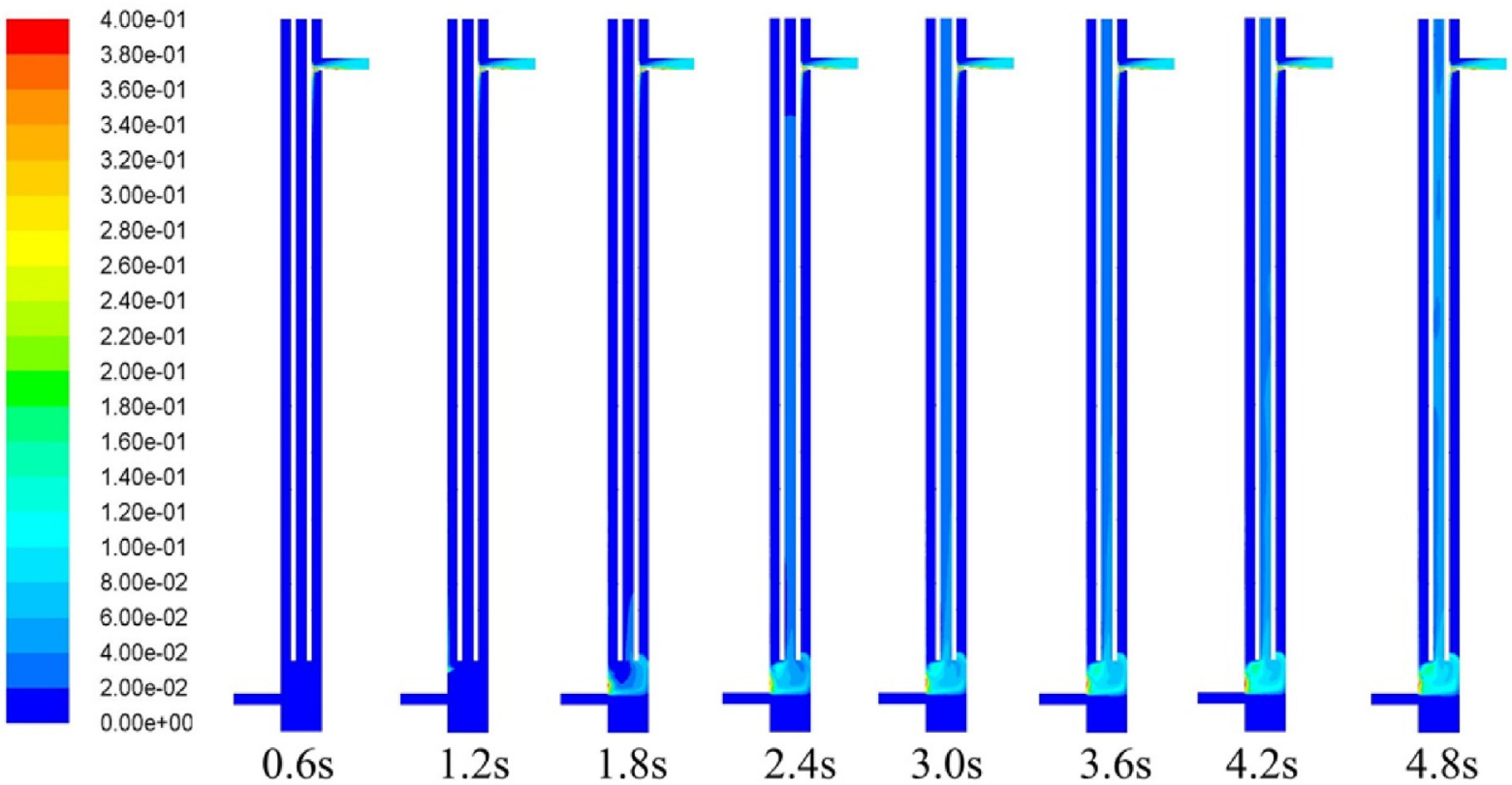

Fig. 12. Water phase distribution for $1.5 \mathrm{~m}^{3} / \mathrm{h}$ water inlet flow rate under different time.

\section{Oil volume fraction}
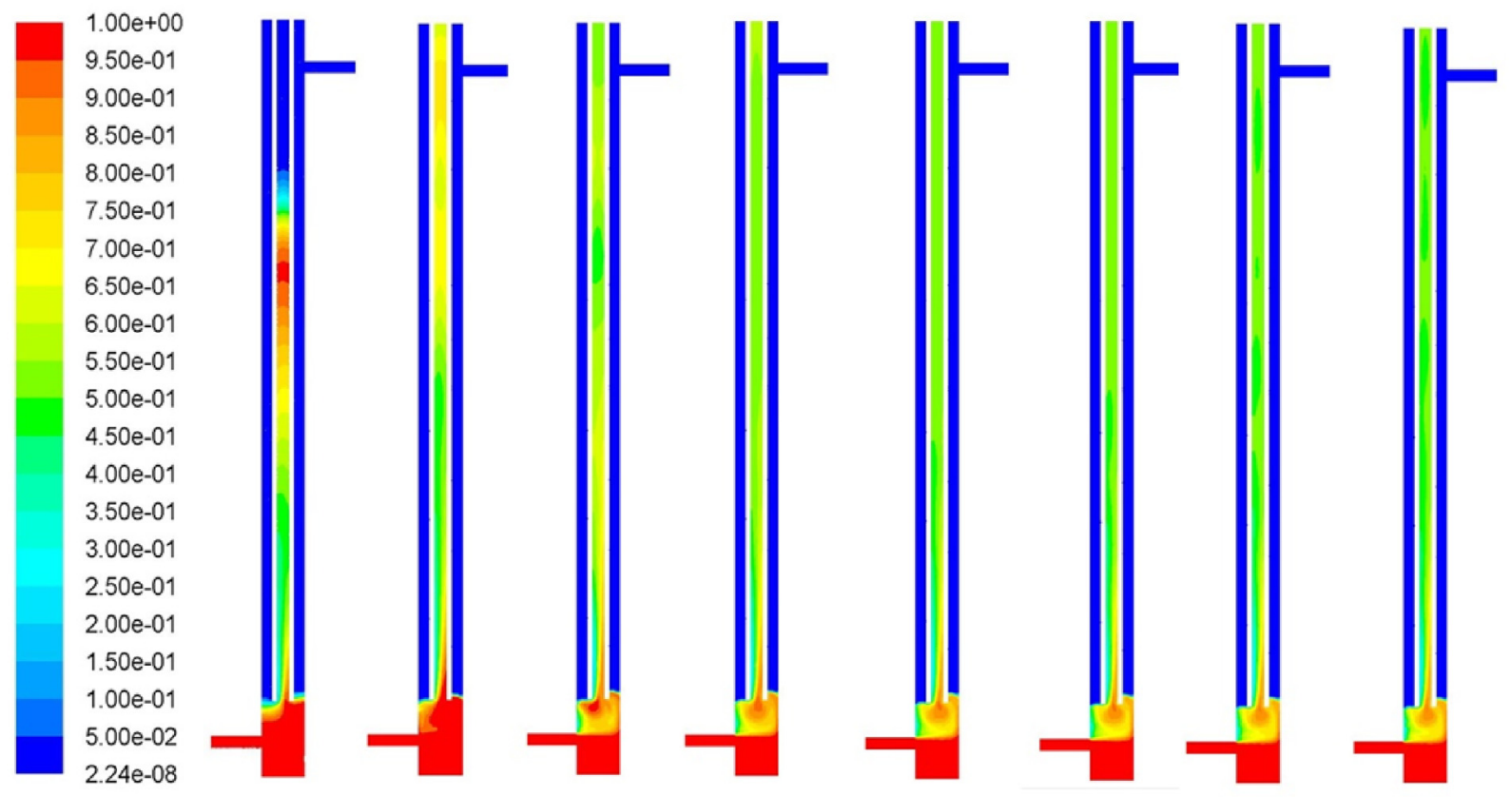

$0.6 \mathrm{~s}$

$1.2 \mathrm{~s}$

$1.8 \mathrm{~s}$

$2.4 \mathrm{~s}$

$3.0 \mathrm{~s}$

$3.6 \mathrm{~s}$

$4.2 \mathrm{~s}$

$4.8 \mathrm{~s}$

Fig. 13. Oil phase distribution for $1.5 \mathrm{~m}^{3} / \mathrm{h}$ water entrance flow rate under different time.

relationship with the turbulent energy dissipation rate during a continuous phase (Zhang and $\mathrm{Xu}, 2016$; Liu et al., 2018).

$d_{\text {sauter }}=\frac{d_{1}^{3}+d_{2}^{3}+d_{3}^{3}+\ldots}{d_{1}^{2}+d_{2}^{2}+d_{3}^{2}+\ldots}$

As shown in Fig. 7, as the inlet flow rate of the water increases from 0.6 to $2.0 \mathrm{~m}^{3} / \mathrm{h}$, the mean Sauter diameter at the spray entrance gradually decreases from 103 to $17 \mu \mathrm{m}$, which is reasonable. As stated above, in an air-blast-atomizer, the volume flow rate ratio of water and air is fixed. With an increase in the water flow rate, the air volume flow rate correspondingly increases, bringing about a larger velocity for a fixed gas chamber. This phenomenon results in a stronger shearing effect, and smaller water droplets consequentially appear.

Under the pressure conditions applied in the experiment, when droplets exit the atomizer and enter the spray entrance under the impact of gravity, they start to coalescence at the bottom of the nozzle. As shown in Fig. 8, when the inlet water flow rate is $1.5 \mathrm{~m}^{3} / \mathrm{h}$ upstream of the atomizer, although the diameter of the inlet droplets is mostly distributed within the $10^{-5} \mathrm{~m}$ scale, the diameter near the spray entrance nozzle enlarges to the millimeter scale, and the total scale of all 
Oil volume fraction
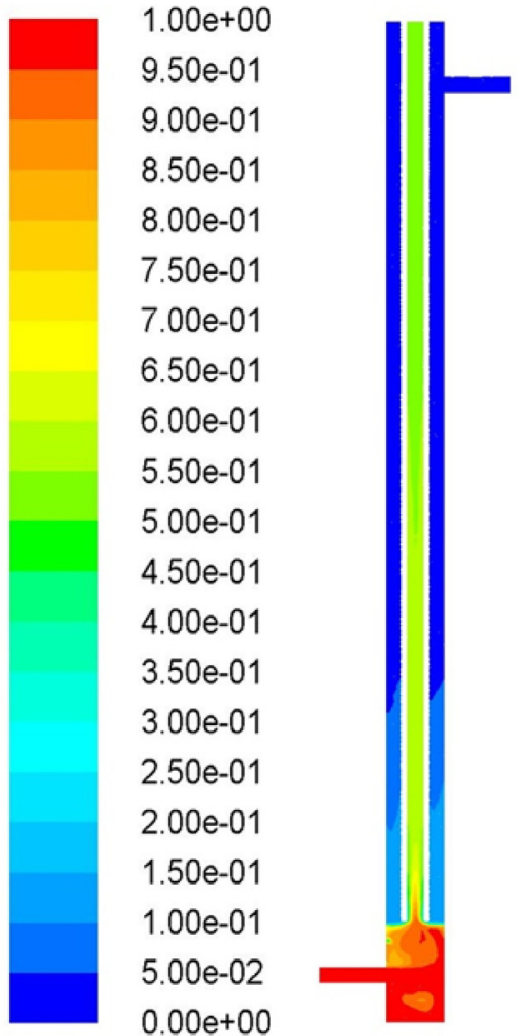

$0.6 \mathrm{~m}^{3} / \mathrm{h}$

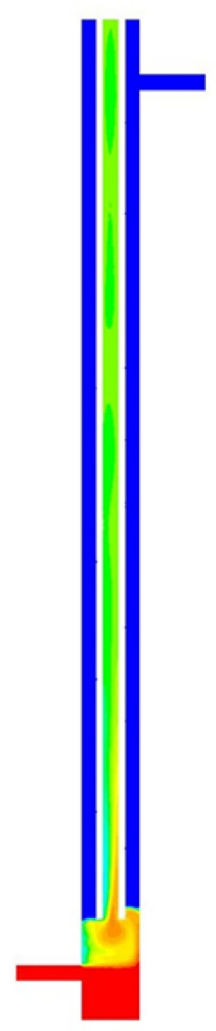

$1.5 \mathrm{~m}^{3} / \mathrm{h}$

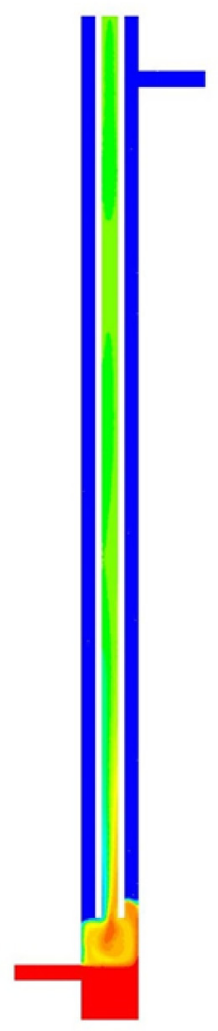

$2.0 \mathrm{~m}^{3} / \mathrm{h}$
Photo

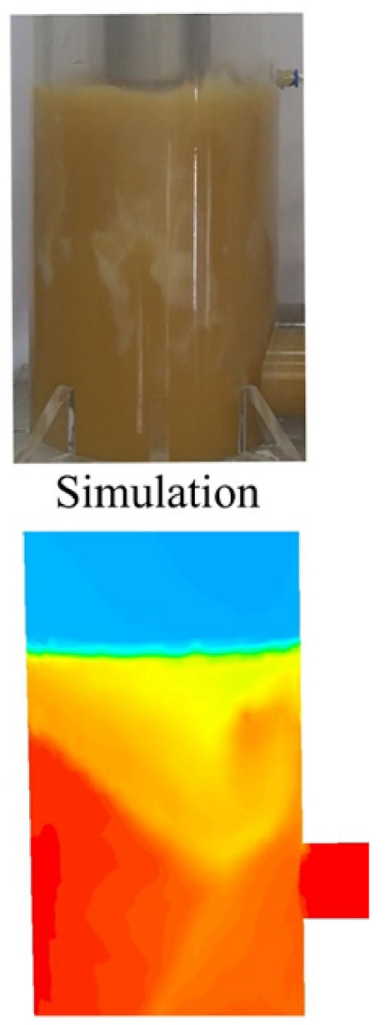

$0.6 \mathrm{~m}^{3} / \mathrm{h}$

Fig. 14. Oil phase distribution under various water entrance flow rates.

Water volume fraction

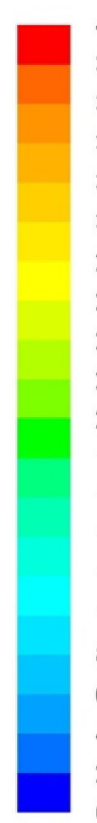

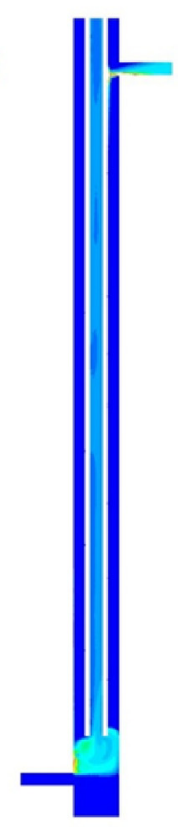

$0.6 \mathrm{~m}^{3} / \mathrm{h} \quad 1.5 \mathrm{~m}^{3} / \mathrm{h}$

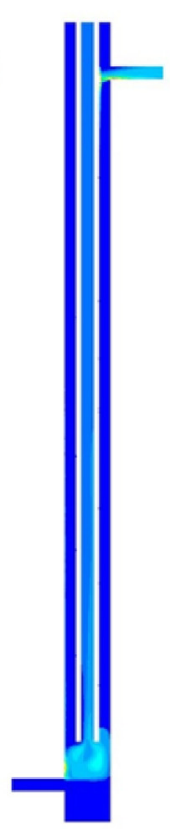

$2.0 \mathrm{~m}^{3} / \mathrm{h}$
Air volume fraction

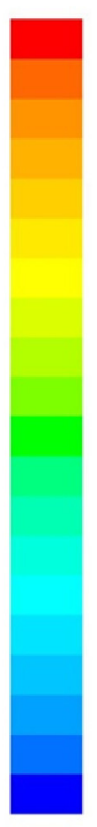

$$
\begin{aligned}
& 1.00 \mathrm{e}+00 \\
& 9.50 \mathrm{e}-01 \\
& 9.00 \mathrm{e}-01 \\
& 8.50 \mathrm{e}-01 \\
& 8.00 \mathrm{e}-01 \\
& 7.50 \mathrm{e}-01 \\
& 7.00 \mathrm{e}-01 \\
& 6.50 \mathrm{e}-01 \\
& 6.00 \mathrm{e}-01 \\
& 5.50 \mathrm{e}-01 \\
& 5.00 \mathrm{e}-01 \\
& 4.50 \mathrm{e}-01 \\
& 4.00 \mathrm{e}-01 \\
& 3.50 \mathrm{e}-01 \\
& 3.00 \mathrm{e}-01 \\
& 2.50 \mathrm{e}-01 \\
& 2.00 \mathrm{e}-01 \\
& 1.50 \mathrm{e}-01 \\
& 1.00 \mathrm{e}-01 \\
& 5.00 \mathrm{e}-02 \\
& 0.00 \mathrm{e}+00
\end{aligned}
$$
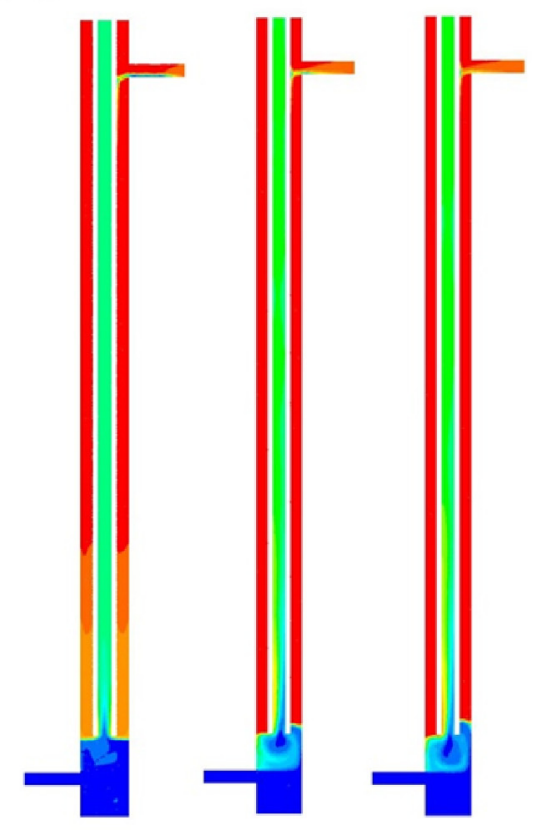

$0.6 \mathrm{~m}^{3} / \mathrm{h} \quad 1.5 \mathrm{~m}^{3} / \mathrm{h} \quad 2.0 \mathrm{~m}^{3} / \mathrm{h}$

Fig. 15. Water and air volume fraction distribution in various operating conditions. 
Mixture viscosity, Pa.s

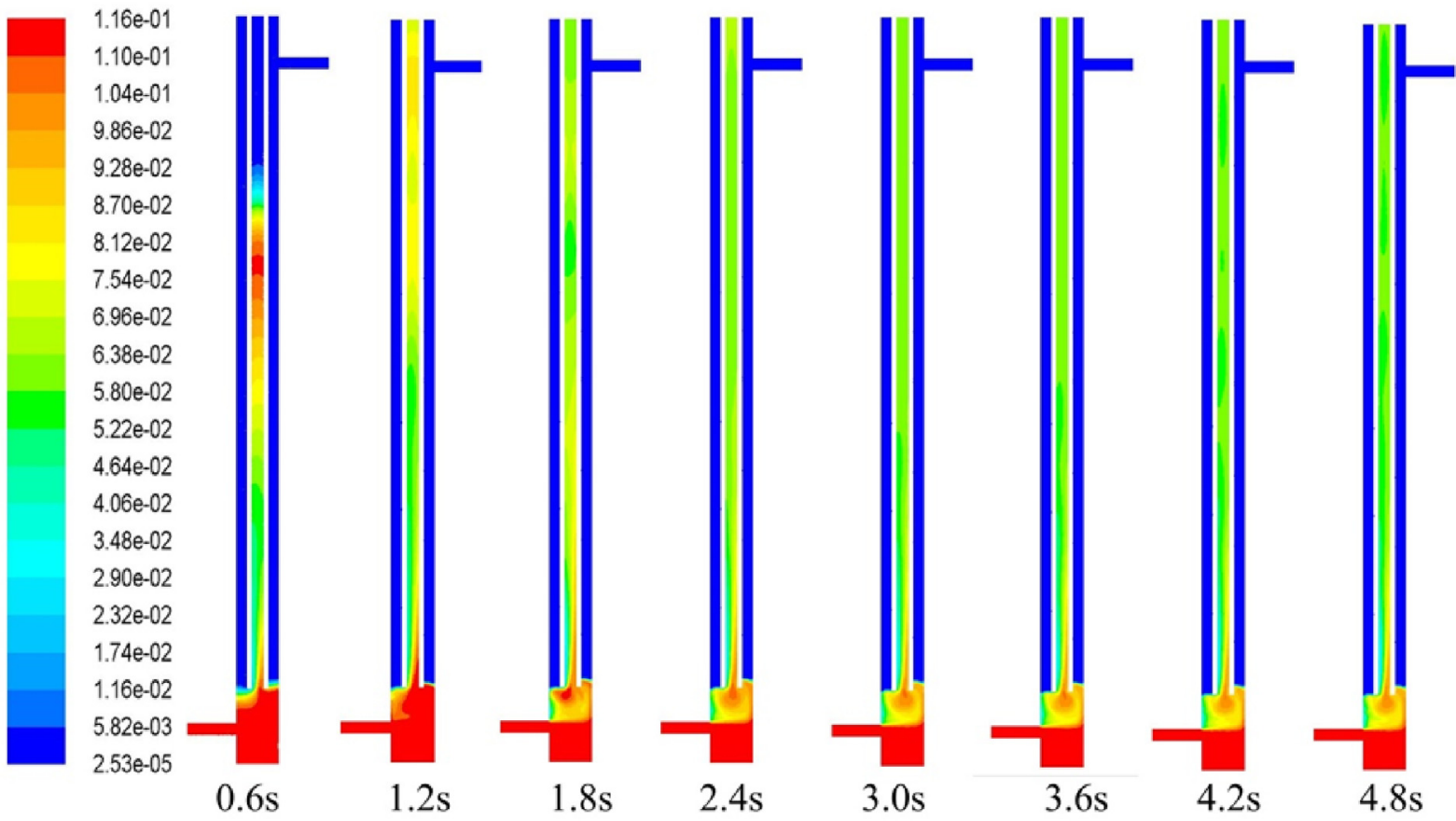

Fig. 16. Mixture viscosity distribution under different time for $1.5 \mathrm{~m}^{3} / \mathrm{h}$ water entrance flow rate.

\section{Mixture viscosity, Pa.s}
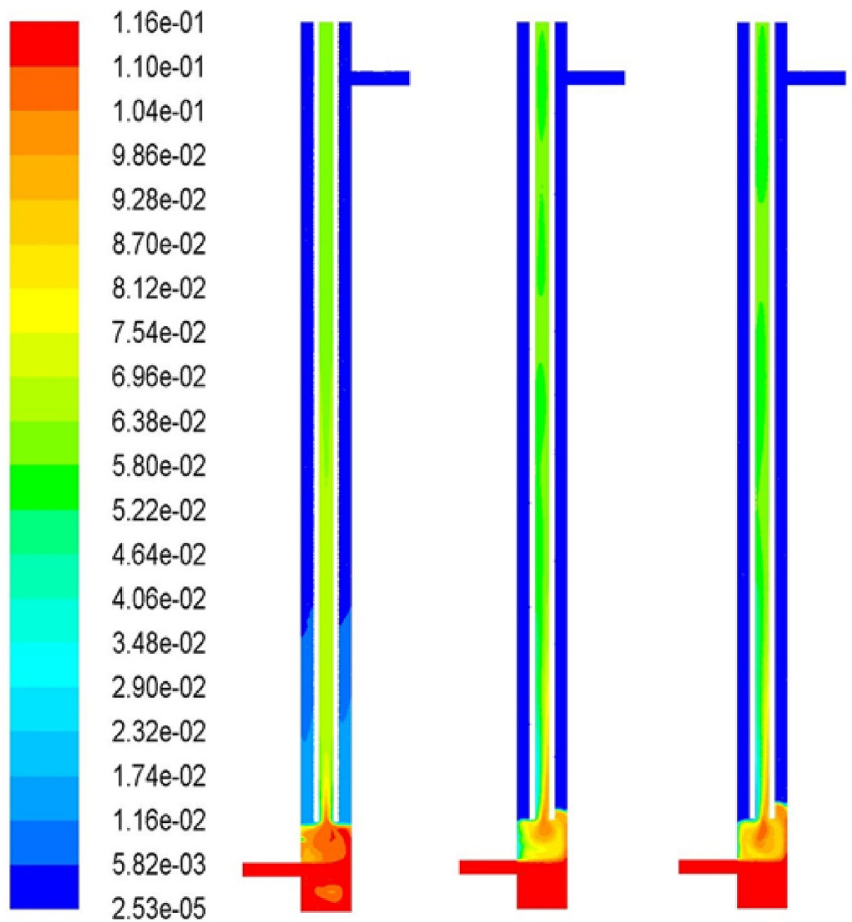

\section{$0.6 \mathrm{~m}^{3} / \mathrm{h}$}

$1.5 \mathrm{~m}^{3} / \mathrm{h}$

Fig. 17. Viscosity distribution for various cases.

water droplets increases, which is due to the influence of the velocity and gravity. Water droplets of a small diameter cannot exist long and coalescence immediately. In addition, a water phase begins to gather at the bottom of the nozzle, flowing into the annulus casing pipe.

As the water flows into the annulus casing, the droplets coalesce

further. Fig. 9 shows the water droplet diameter for a $1.5 \mathrm{~m}^{3} / \mathrm{h}$ inlet flow rate at various moments of $0.6 \mathrm{~s}-4.8 \mathrm{~s}$ with a $0.6 \mathrm{~s}$ time interval. In the figure, the red color represents large droplets whereas the blue color represents small droplets. The sizes of the droplets range from $3 \mu \mathrm{m}$ to $11.1 \mathrm{~mm}$. In the annulus casing, a water droplet near the left wall (which is at the side of the oil injection nozzle) becomes larger than that on the equivalent area on the right after $1.2 \mathrm{~s}$. This means that a considerable number of water droplets transit, bypassing the steel lifting pipe and hitting the right side of the plexiglass wall, where they gather into a liquid film and flow downward. The gathered water flows down along the wall until it meets the oil. The water then enters the previous oil zone, where it gradually gathers, and the volume fraction increases (as is shown in Fig. 12), which in turn promotes the water droplets to collide and increase in size. By contrast, inside the steel lifting pipe, droplets start to appear at $1.8 \mathrm{~s}$ and gradually fill in the entire pipe. Compared with their size in the casing pipe, the droplets in the lifting pipe are smaller. This is due to a higher velocity in the lifting pipe bringing about a stronger shearing effect and relative uniform blending among phases. At $3.6 \mathrm{~s}$, the droplet size begins stabilize and changes only slightly.

As the boundary conditions change, as indicated in Fig. 10, the droplet size distribution differs according to the water entrance flow rate. Hinze (1955) proposed the idea that the droplet size has an influence on the shear velocity during a continuous phase in a pipe flow. As shown in Fig. 10, in a lifting pipe, the water droplet size decreases as mixture velocity increases, and the mixture velocity has a positive correlation with the inlet water flow rates. However, Hinze's proposal is not in accordance with the droplets found in the casing. In the annulus space at the upper side of the initial air-oil interface, the water droplet size increases with the inlet water flow rate, particularly for the oil entrance nozzle side. As the reason for this, as the water and oil entrance velocities increase, resistance preventing air from joining with the water droplets as they flow down to the steel lifting pipe increases. This promotes an easier collision of the water droplets. Moreover, as the water droplets are injected into the nozzle more quickly, more of the water phase flow bypasses the lifting pipe and hits the casing wall, and thus more of the gathered water appears as a film. For water droplets in 

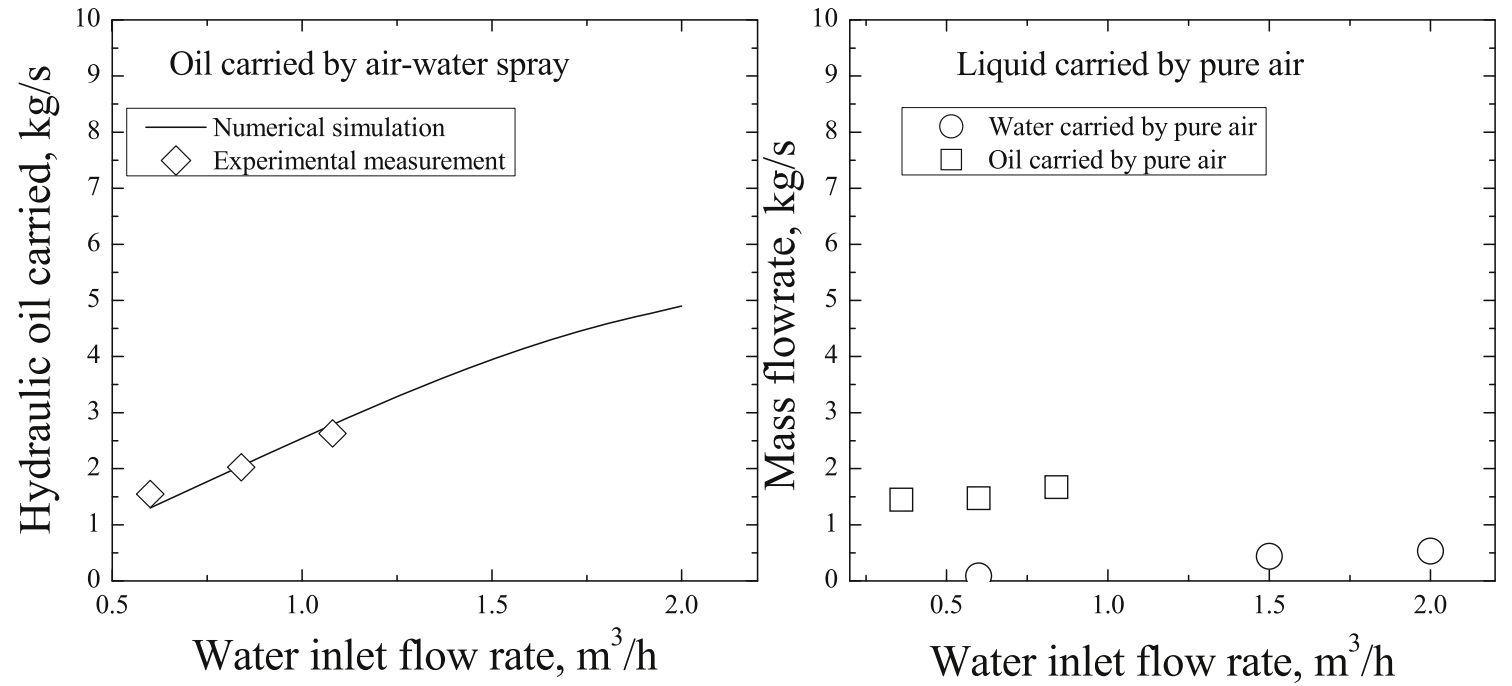

Fig. 18. Oil and water carrying at the exit of steel lifting pipe both numerically and experimentally.

the blending zone prior to entering the lifting pipe, an action and a counter action occur between the water droplet breakup and the coalescence brought about by a circular flow. Under this effect, the distribution of the droplet sizes is irregular compared with that in a lifting pipe.

Fig. 11 shows further data on the water droplet size distribution, illustrating the volume fraction distribution for a certain droplet size under various boundary conditions. The curves can be divided into three parts for most of the conditions. The peak values between 1.5 and $3 \mathrm{~mm}$ represent droplets in the lifting pipe. The peak at approximately $5 \mathrm{~mm}$ shows water droplets in the casing. The peak at approximately $8 \mathrm{~mm}$ corresponds with water in the blending zone and the water film in the upper part of the oil entrance nozzle. For a water inlet flow rate of $0.6 \mathrm{~m}^{3} / \mathrm{h}$, owing to the relatively low velocity, the droplet sizes in the casing and lifting pipe do not differ to the extent that the peaks merge at a wider peak visually. In addition, as an obvious trend, the droplet size corresponding with the peak of the volume fraction decreases as the entrance flow rate increases.

\subsection{Phase blending behavior}

The blending behavior is directly characterized by the phase distribution. Figs. 12 and 13 show the water and oil phase distributions for a water inlet flow rate of $1.5 \mathrm{~m}^{3} / \mathrm{h}$ at various moments. As described in section 4.1, initially, the water phase under the influence of gravity starts to gather at the bottom of the spray entrance nozzle. It then hits the steel lifting pipe wall and some of it flows downward. Most other parts of the flow bypass the steel lifting pipe and gather in the upper part of the oil entrance nozzle. Later, at $1.8 \mathrm{~s}$, the water phase invades the initial oil zone and flows with the oil phase in the form of a circular flow. The water flow behavior is similar with the pathline shown in Fig. 6. At the same time, the water begins to appear in the lifting pipe. At $3.0 \mathrm{~s}$, the water phase distribution stabilizes at the blending zone near the entrance of steel lifting pipe, whereas the water concentration continuous to increase until $4.8 \mathrm{~s}$ in the lifting pipe.

As with the oil phase, Fig. 13 shows the phase distribution at different moments corresponding with the water phase. Initially, the oil lifting is similar with the gas lifting. In the steel lifting pipe, after a block of pure oil is pumped up to the exit, blending occurs between the oil and gas phases. Oil fraction zones of 0.75 and 0.45 alternatively appear until the water droplets enter the lifting pipe, which is similar with a slug flow (Bellarby, 2009) and is not beneficial for heavy crude lifting. As the water droplets begin to mix with the oil near the entrance of the lifting pipe, the oil fraction decreases. The performance of the spray blending is better than that of pure gas lifting. At $2.4 \mathrm{~s}$, the existing water droplets gradually stabilize the volume fraction of the oil phase in the lifting pipe. The oil fraction stabilizes at 0.5, whereas the air and water phase fractions stable at 0.45 and 0.05 , respectively.

For the blending behavior under different operating conditions, Fig. 14 shows a comparison of the phase distribution near the Plexiglass wall for the experiment observation and numerical simulation at a water entrance flow rate of $0.6 \mathrm{~m}^{3} / \mathrm{h}$. In comparing the numerical simulation with the experiment observation, an interface can be seen to exist. Immediately after the interface, water invades the oil in the form of a liquid film, which appears as the white liquid in the photograph. Correspondingly, the phase concentration decreases slightly during the numerical simulation. In addition, the shape of the contour line is similar with that in the photograph, validating the numerical simulation to a certain extent.

Figs. 14 and 15 show the phase distributions at water entrance flow rates of $0.6,1.5$, and $2.0 \mathrm{~m}^{3} / \mathrm{h}$. Blending between the oil and spray mainly occurs in the section between the oil entrance nozzle and the lifting pipe entrance, which is called the blending zone herein. The oil fraction is high under a water entrance flow rate of $0.6 \mathrm{~m}^{3} / \mathrm{h}$ in the blending zone. Under other conditions, oil in the blending zone is invaded by a spray near the casing wall. In addition, the volume fraction is reduced. The shape of the oil fraction of lower than 0.75 looks similar to a hook, which can be attributed to the anti-clock circular flow of the oil phase joining with the discrete bubbles and water droplets. The bubbles and droplets penetrating the blending zone are carried by the continuous oil phase and become mixed before entering the lifting pipe. At a small entrance flow rate $\left(0.6 \mathrm{~m}^{3} / \mathrm{h}\right)$, owing to the low velocity, bubbles and droplets cannot penetrate too far into the blending zone, blending only exists near the interface, and the circular flow is low. In the remaining cases, the spray velocity is sufficiently high to achieve an upper boundary of the oil entrance nozzle. The circular flow induced by the oil phase is also strong. Consequently, considerable bubbles and droplets follow the circular oil flow and become mixed in the blending zone. However, a difference occurs for the air and water distribution in the blending zone when considering a hollow core appearing around the hook-shaped route. This can be attributed to the variation in density and surface tension between air and water. A small density and larger surface tension with oil prevent air from entering the hollow core surrounded by the hook-shaped area. In addition, the interface between air and liquid for the spray injection side increases, which is reasonable. The air continuously rushes into the oil phase owing to large disturbances and fractures of the bubbles. The water film and water droplets invade the oil phase as well, bringing about a larger disturbance. 

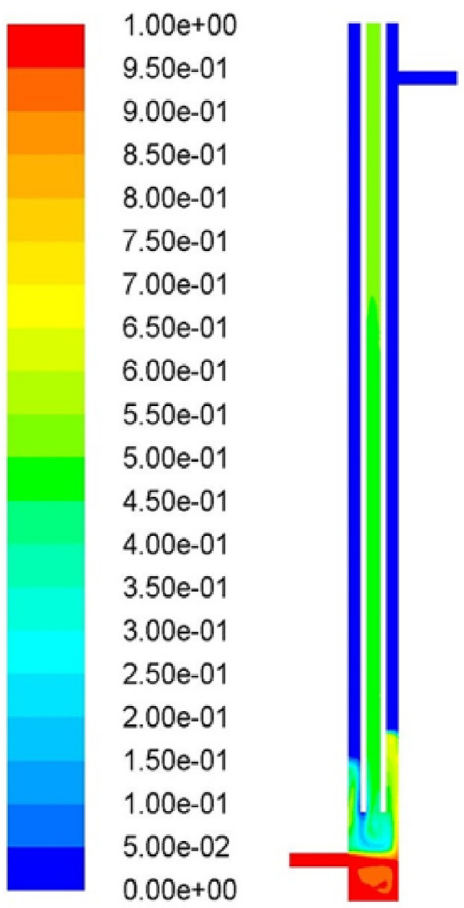

Volume fraction

Oil

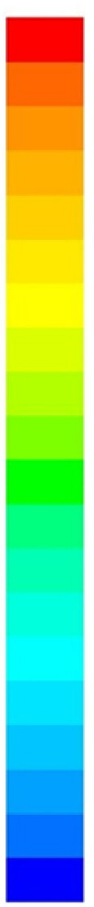

$1.11 \mathrm{e}-02$
$1.05 \mathrm{e}-02$
$9.99 \mathrm{e}-03$
$9.43 \mathrm{e}-03$
$8.88 \mathrm{e}-03$
$8.32 \mathrm{e}-03$
$7.77 \mathrm{e}-03$
$7.21 \mathrm{e}-03$
$6.66 \mathrm{e}-03$
$6.10 \mathrm{e}-03$
$5.55 \mathrm{e}-03$
$5.00 \mathrm{e}-03$
$4.44 \mathrm{e}-03$
$3.89 \mathrm{e}-03$
$3.33 \mathrm{e}-03$
$2.77 \mathrm{e}-03$
$2.22 \mathrm{e}-03$
$1.66 \mathrm{e}-03$
$1.11 \mathrm{e}-03$
$5.55 \mathrm{e}-04$
$0.00 \mathrm{e}+00$

Global droplet size, $\mathrm{m}$

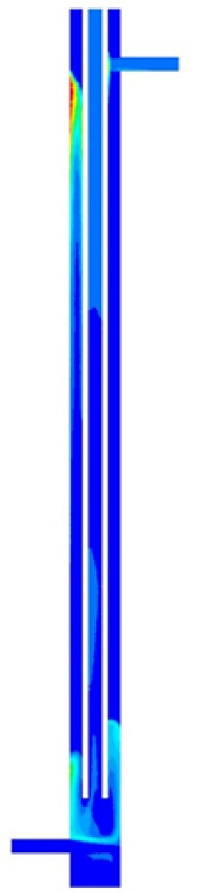

Water

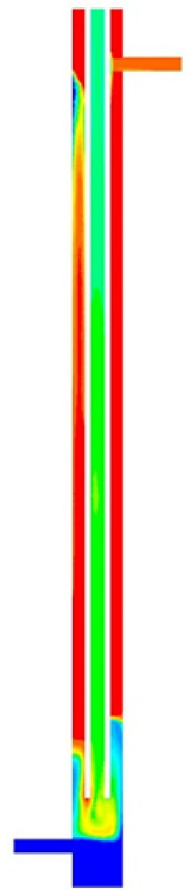

Air
$1.54 \mathrm{e}-03$

$1.51 \mathrm{e}-03$

$1.48 \mathrm{e}-03$

$1.45 \mathrm{e}-03$

$1.42 \mathrm{e}-03$

$1.39 \mathrm{e}-03$

$1.36 \mathrm{e}-03$

$1.33 \mathrm{e}-03$

$1.30 \mathrm{e}-03$

$1.27 \mathrm{e}-03$

$1.24 \mathrm{e}-03$

$1.21 \mathrm{e}-03$

$1.18 \mathrm{e}-03$

$1.15 \mathrm{e}-03$

$1.12 \mathrm{e}-03$

$1.09 \mathrm{e}-03$

$1.06 \mathrm{e}-03$

$1.03 \mathrm{e}-03$

$1.00 \mathrm{e}-03$

$9.71 \mathrm{e}-04$

9.41e-04

Lifting droplet size, $\mathrm{m}$
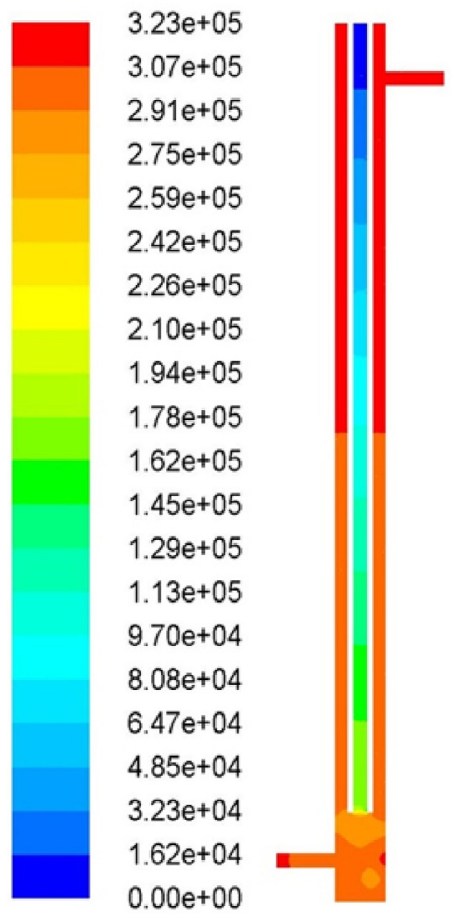

Pressure, $\mathrm{Pa}$
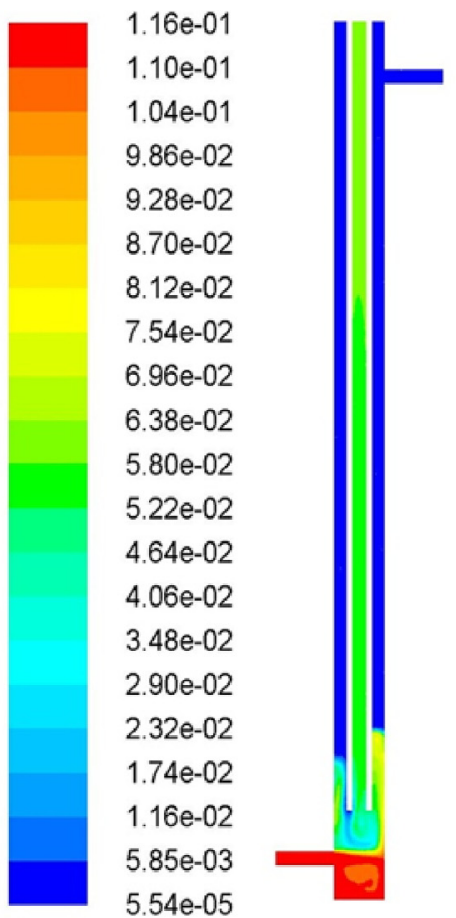

Mixture viscosity, Pa.s

Fig. 19. Blending properties under high pressure drop conditions.

Although a difference exists in the blending zone, phases have been blended well in the lifting pipe.

\subsection{Viscosity distribution and oil carrying ability}

Two of the most important characteristics of atomized blending properties are the viscosity reduction and oil carrying capability. Based on the discussion above, this subsection provides a deep investigation into this area.

For a fixed water flow rate at the entrance, the evolution of the mixture viscosity over time should first be focused upon. Fig. 16 shows the change in viscosity over time under a water entrance flow rate of $1.5 \mathrm{~m}^{3} / \mathrm{h}$. As discussed above, the behavior of the flow domain is similar to the lifting of pure gas at before $1.2 \mathrm{~s}$. At this moment, relatively high mixture viscosities of 90 and $70 \mathrm{mPa}$ s occur alternatively until $3.0 \mathrm{~s}$, at which the water droplets fill the lifting pipe, and the mixture 
viscosity is diminished to $58 \mathrm{mPa}$, which is $50 \%$ of the pure oil viscosity. Moreover, the distribution of the mixture viscosity is more uniform than under gas-lifting conditions, and the viscosity reduction of air-blast-atomization is ideal.

Fig. 17 depicts steady viscosity distribution of flow domain under various inlet water flow rates. As water entrance flow rate increase, average viscosity standard changes little in most part of the central steel pipe. And the high viscosity part at entrance of central steel pipe gradually move to one side and the viscosity value turns to be slashed. Moreover, viscosity under steel lifting pipe gradually falls down which means oil-water-gas get fully blended. In all, viscosity reduction of airblast-atomize is ideal.

Regarding the oil carrying abilities, Fig. 18 shows the oil and water carrying at the exit of the steel lifting pipe both numerically and experimentally. The image on the left side of Fig. 18 compares the measurements taken during the experiment and the numerical simulation. The curve indicates the simulation whereas the scatter plot represents the measurements. As the image indicates, the oil carrying capacities as shown numerically and experimentally are in good agreement, which also validates the numerical simulation. As shown in the total trend, as the water flow rate increases at the entrance, the amount of oil lifted at the exit increases concurrently. Moreover, comparing the experiment data on the left and right sides, which show oil carried by an air-water spray and pure air, it is clear that oil carried by the air-water spray is larger in amount than that of pure air, and the difference increases as water flow rate at the inlet (for pure air conditions, the inlet air flow rate is 10 times that of the corresponding inlet water flow rate) increases. Under $0.6 \mathrm{~m}^{3} / \mathrm{h}$ water inlet flow rate conditions, oil lifted is $8 \%$ higher for spray blending than gas lifting. As water inlet flow rate increases to $0.84 \mathrm{~m}^{3} / \mathrm{h}$, the variance increases to $16 \%$. This proves that atomization blending achieves a better performance than pure air lifting.

\subsection{Atomization blending properties under high-pressure drop environments}

The studies above were conducted under an ordinary pressure drop at the $10 \mathrm{kPa}$ scale. In the heavy crude extraction on-site, the pressure drop scale is larger. Thus, a blending investigation under a larger pressure drop is essential for further investigation. The discussion above indicates that the numerical simulation is reliable for the simulation of the blending behavior. Hence, the blending properties were investigated under larger pressure drop conditions through a numerical simulation.

Fig. 19 shows the blending properties under conditions of a large pressure drop. The total pressure drop from the entrance to exit is $380 \mathrm{kPa}$. Under the conditions of a high pressure drop, a small portion of the water phase injected into the nozzle hits the central steel pipe and gathers into a water film, and most of the water droplets hit the wall at the upper part of the oil entrance nozzles, gathering in a water film formation. As with the water droplet size, although the local size diameter in the lifting pipe decreases owing to a strong shearing effect under a continuous phase, the diameter in the annulus space in the casing pipe remains large, which means that a water film appears and gathers at the casing wall. For the oil phase, a considerable portion of oil rushed into the annulus casing pipe with a volume fraction of $0.3-0.6$. In the lifting pipe, the oil volume fraction is relatively distributed at 0.5 . As with the air phase, it gathers around the hook-shaped route with a volume fraction of 0.7 near the lifting pipe entrance. Based on the phase distribution described above, the phase blending is more severe.

In terms of the viscosity distribution, although the mixture viscosity in the steel lifting pipe is not uniform, the total value is approximately $60 \mathrm{mPa} \mathrm{s}$, half that of the oil viscosity. In addition, the oil carried outside is $11.8 \mathrm{~kg} / \mathrm{s}$, which is proportionate with the augmenting of the inlet flow rates. Each of these aspects validates the atomization blending performance under large pressure drop conditions.

\section{Conclusions}

In this study, a thorough investigation into the atomization blending properties of heavy crude extraction was conducted both experimentally and numerically. In the experimental portion of the study, after a systematic dimensionless analysis, an investigation was conducted on the equipment shearing in the same sized area as an on-site production. In the numerical simulation, an Eulerian multiphase model, along with a population balance model and an RNG $k-\varepsilon$ turbulent model, was applied for a detailed look into the blending behavior. The following conclusions were drawn:

Under a 1:10 water-air flow rate ratio spray, phase blending mainly occurs at locations between the oil injection nozzle and the entrance of the lifting wall, which is also called the blending zone. Oil could be uniformly distributed in the lifting pipe with a volume fraction of 0.5-0.6.

Water droplets gathered in the annulus casing wall in the form of a water film and flowed downward. The droplet size in the lifting pipe shears similar trends with the pipe flow, while the droplet size in the blending zone decreases slightly as inlet flow rate increases.

Atomization blending can reduce the oil viscosity. The experiment results prove the flow properties well, and the numerical simulation shows that the viscosity can be reduced $50 \%$ through atomization blending.

The atomization blending method has a better oil carrying ability compared with gas lifting. Under $0.6 \mathrm{~m}^{3} / \mathrm{h}$ water inlet flow rate, spray blending lifts $8 \%$ more heavy crude than gas lifting. As the inlet flow rate increases, the difference between the atomization blending and pure air lifting becomes significant. Moreover, the atomization blending performs well under high pressure drop conditions.

\section{Acknowledgement}

The authors gratefully acknowledge that the work described here is financially supported by Exploration and Development Area Optimization and Object Appraisal of Continental Shale Oil in Typical Basins in China (No.2017ZX05049) and National Natural Science Foundation of China (No. 51779243).

\section{Appendix A. Supplementary data}

Supplementary data to this article can be found online at https:// doi.org/10.1016/j.petrol.2019.106494.

\section{References}

Bellarby, J., 2009. Well Completion Design, vol. 56 Elsevier, Amsterdam, The Netherlands978-0-444-53210-7.

Du, Y., Guan, L., 2004. Field-scale polymer flooding: lessons learnt and experiences gained during past 40 years. In: SPE International Petroleum Conference in Mexico. Society of Petroleum Engineers. https://doi.org/10.2118/91787-MS.

Gao, C.H., 2011. Scientific research and field applications of polymer flooding in heavy oil recovery. J. Pet. Explor. Prod. Technol. 1, 65-70. https://doi.org/10.1007/ s13202-011-0014-6.

Ghannam, M.T., Hasan, S.W., Abu-Jdayil, B., Esmail, N., 2012. Rheological properties of heavy \& light crude oil mixtures for improving flow ability. J. Pet. Sci. Eng. 81, 122-128. https://doi.org/10.1016/j.petrol.2011.12.024.

Guet, S., Ooms, G., 2006. Fluid mechanical aspects of the gas-lift technique. Annu. Rev. Fluid Mech. 38, 225-249. https://doi.org/10.1146/annurev.fluid.38.061505. 093942.

Guo, K., Li, H., Yu, Z., 2016. In-situ heavy and extra-heavy oil recovery: a review. Fuel 185, 886-902. https://doi.org/10.1016/j.fuel.2016.08.047.

Hasan, S.W., Ghannam, M.T., Esmail, N., 2010. Heavy crude oil viscosity reduction and rheology for pipeline transportation. Fuel 89, 1095-1100. https://doi.org/10.1016/j. fuel.2009.12.021.

Hinze, J.O., 1955. Fundamentals of the hydrodynamic mechanism of splitting in disper sion processes. AIChE J. 1, 289-295. https://doi.org/10.1002/aic.690010303.

Hirleman, E.D., Oechsle, V., Chigier, N.A., 1984. Response characteristics of laser diffraction particle size analyzers: optical sample volume extent and lens effects. Opt. Eng. 23, 610-619. https://doi.org/10.1117/12.7973344.

Jagadevan, S., Banerjee, A., Banerjee, C., Guria, C., Tiwari, R., Baweja, M., Shukla, P., 
2018. Recent developments in synthetic biology and metabolic engineering in microalgae towards biofuel production. Biotechnol. Biofuels 11, 185-205. https://doi org/10.1186/s13068-018-1181-1.

Jing, J., Yin, R., Zhu, G., Xue, J., Wang, S., Wang, S.H., 2019. Viscosity and contact angle prediction of low water-containing heavy crude oil diluted with light oil. J. Pet. Sci. Eng. 176, 1121-1134. https://doi.org/10.1016/j.petrol.2019.02.012.

Li, H.Y., Cui, K.X., Jin, L., Jin, L., Wang, L.L., Yu, Bo, 2018a. Experimental study on the viscosity reduction of heavy oil with nano-catalyst by microwave heating under low reaction temperature. J. Pet. Sci. Eng. 170, 374-382. https://doi.org/10.1016/j. petrol.2018.06.078.

Li, X.L., Shi, L.X., Li, H.Z., Liu, P.C., Luo, J., Yuan, Z., 2018b. Experimental study on viscosity reducers for SAGD in developing extra-heavy oil reservoirs. J. Pet. Sci. Eng. 166, 25-32. https://doi.org/10.1016/j.petrol.2018.03.022.

Liao, Y.X., Lucas, D., 2009. A literature review of theoretical models for drop and bubble breakup in turbulent dispersions. Chem. Eng. Sci. 64, 3389-3406. https://doi.org/10. 1016/j.ces.2009.04.026.

Liu, S., Zhang, D., Yang, L.L., Xu, J.Y., 2018. Breakup and coalescence regularity of nondilute oil drops in a vane type swirling flow field. Chem. Eng. Sci. Des. 129, 35-54. https://doi.org/10.1016/j.cherd.2017.10.033.

Luo, F., Cui, H., Dong, S., 2014. Transient measuring method for injection rate of each nozzle hole based on spray momentum flux. Fuel 125, 20-29. https://doi.org/10. 1016/j.fuel.2014.02.011.

Martínez-Palou, R., Lourdes-Mosqueira, M., Zapata-Rendón, B., Mar-Juárez, E., BernalHuicochea, C., Cruz Clavel-López, J., Aburto, J., 2011. Transportation of heavy and extra-heavy crude oil by pipeline: a review. J. Pet. Sci. Eng. 75, 274-282. https://doi. org/10.1016/j.petrol.2010.11.020

Rangel-German, E.R., Schembre, J., Sandberg, C., Kovscek, A.R., 2004. Electrical-heating assisted recovery for heavy oil. J. Pet. Sci. Eng. 45, 213-231. https://doi.org/10. 1016/j.petrol.2004.06.005.

Shuba, E.S., Kifle, D., 2018. Microalgae to biofuels: 'Promising' alternative and renewable energy, review. Renew. Sustain. Energy Rev. 81, 743-755. https://doi.org/10.1016/ j.rser.2017.08.042.

Subramanian, D., Wu, K., Firoozabadi, A., 2015. Ionic liquids as viscosity modifiers for heavy and extra-heavy crude oils. Fuel 143, 519-526. https://doi.org/10.1016/j.fuel. 2014.11.051.

Wang, C., Huang, B., Wang, G., Zhang, M.D., Ding, N., 2017. Unsteady pressure fluctuation characteristics in the process of breakup and shedding of sheet/cloud cavitation. Int. J. Heat Mass Transf. 114, 769-785. https://doi.org/10.1016/j ijheatmasstransfer.2017.06.005.

Wu, P.K., Ruff, G., Faeth, G., 2006. Primary breakup in liquid/gas mixing layers. Atomization Sprays 1, 421-440. http://doi.org/10.1615/AtomizSpr.v1.i4.50.

Xia, Y., Khezzzar, L., Alshehhi, M., Hardalupas, Y., 2017. Droplet size and velocity characteristics of water-air impinging jet atomizer. Int. J. Multiph. Flow 94, 31-43. https://doi.org/10.1016/j.ijmultiphaseflow.2017.04.014.

Yang, Z.P., 2017. Simulation and Optimization of the Thick Oil in the Well, vol. 2017 Daqing: Northeast petroleum university.

Zhang, J., Xu, J.Y., 2016. Rheological behavior of oil and water emulsions and their flow characterization in horizontal pipes. Can. J. Chem. Eng. 94, 324-331. https://doi. org/10.1002/cjce.22377. 